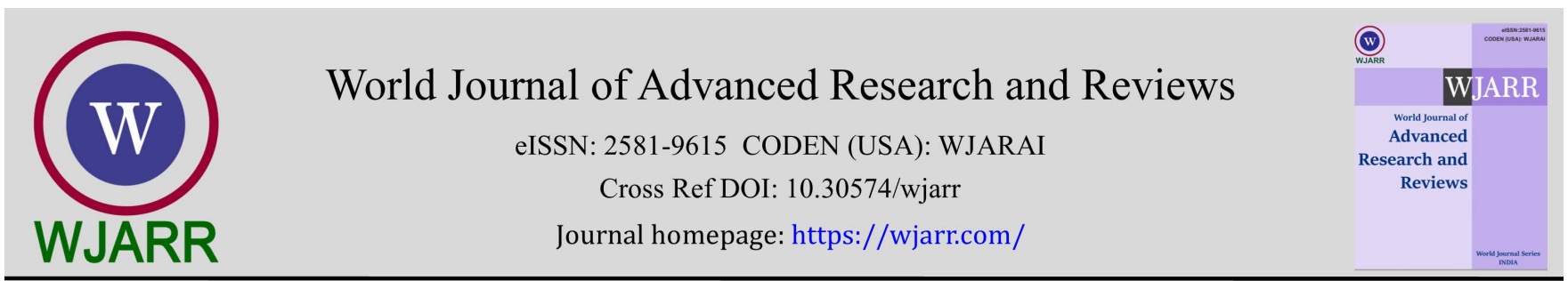

(RESEARCH ARTICLE)

\title{
Bio-inspired central pattern generator for development of Cartesian motor skills for a Quadruped Robot
}

\author{
Farhad Asadi *, Mahdi Khorram and S Ali A Moosavian \\ Department of Mechanical Engineering, K. N. Toosi University of Technology, Center of Excellence in Robotics and Control, \\ Advanced Robotics \& Automated Systems (ARAS) Lab Affiliation, Tehran, Iran.
}

World Journal of Advanced Research and Reviews, 2021, 12(01), 066-085

Publication history: Received on 23 August 2021; revised on 29 September 2021; accepted on 01 October 2021

Article DOI: https://doi.org/10.30574/wjarr.2021.12.1.0485

\begin{abstract}
Central Pattern Generator (CPG) plays a significant role in the generation of diverse and stable gaits patterns for animals as well as controlling their locomotion. The main contributions of this paper are the ability to develop the Cartesian motor skills and coordinating legs of the quadruped robot for gait adaption and its nominal characteristics with CPG approach. Primary, a predefined relationship between an excitation signal and essential parameters of the CPG design is programmed. Next, the coordinated oscillator's rhythmic patterns by CPG and accordingly output gait diagrams for each foot of the robot are attained. Then, these desirable features such as predictive modulation and programming the gait event sequences including leg-lifting sequences and step length, duration of the time of each footstep within a gait, coordination of swing and stance phases of all legs are calculated in terms of different spatio_temporal vectors. Furthermore, a novel Cartesian footstep basis function is designed based on the robot characteristics and consequently, the associated spatio-temporal vectors can be inserted to it, which caused to spanning the space of possible gait timing in Cartesian space. Next, Cartesian footstep planner can be computed the swing foot trajectories in workspace along movement axes and then according to these footholds and feet placement, ZMP (Zero Moment Point) reference trajectory will be calculated and obtained. Therefore, COG (Center of Gravity) trajectory can be computed by designing a preview controller on the basis of the desired ZMP trajectory. Finally, to demonstrate the effectiveness of the proposed algorithm, it is implemented on a quadruped robot on both simulation or experimental implementations and the results are compared and discussed with other references.
\end{abstract}

Keywords: Central Pattern Generator; Gait generation and transition; Essential gaits for quadruped robot; Motor pattern generation; Motor skills

\section{Introduction}

The best definition for the ability of four-legged robot means their capability to produce diverse motor functions such as walking, like other creatures. Four-legged creatures are able to move with rich and diverse speed and gait depending to conditions of the environment. Most researchers, in different fields from biomedical engineering to neuroscience, were investigated these principles and consequently many optimal and intelligence approaches are available for inspiration and using in the robotic system [1]. Principally, animals for achieving to their desired speed change and adjust their gaits continuously [2]. These abilities are due to the central neural networks in their spinal cords, which consists of a network of the neurons that has the ability to produce rhythmic patterns without receiving any feedback $[3,4]$. Since in many works of literature, the existence and functionality of this issue were substantiated, many researchers have proposed an algorithm based on the various abstract modelling of this network. This diversity of modelling is from biophysical and neural network modelling to approximate models such as nonlinear oscillators.

\footnotetext{
* Corresponding author: Farhad Asadi; Email:Farhadasadi1990@gmail.com

Department of Mechanical Engineering, K. N. Toosi University of Technology, Center of Excellence in Robotics and Control, Advanced Robotics \& Automated Systems (ARAS) Lab Affiliation, Tehran, Iran.
} 
The concept of CPG in robotic is interpreted with coupling and organization of oscillator's network, in order to reach to reasonable locomotion patterns [5, 6]. So, in this area for developing the CPG methods, the theory of dynamical systems and signal processing should be applied and developed in order to make functional CPG for motor pattern generation $[7,8]$.

Principally, for generation of different adaptable gaits and also in order to switch between them, a synergetic rhythmic patterns among foots or joints of the robot is necessary $[9,10]$. Some references $[11,12]$, used HOPF oscillator in CPG network and modelled each gait of the robot with determining one predefined matrix. In this approach, each row and column of the matrix is associated to coupling coefficients between the oscillators, and the elements of matrix should be changed for gait adjusting at the desired time. Moreover, the references [13-17], used this coefficient matrix approach with both HOPF and Vander pol oscillator, but in all of these references, the gait of the robot without controlling speed or relative phases between the legs are changed suddenly at the predefined time. Many researchers have studied and classified different coupling approaches in order to achieve to diverse flexible spatial-temporal patterns [18]. In this paper, the CPG must be adaptive and evolution of patterns of the oscillators should be a combination of the intrinsic dynamics of the oscillators and adaptation rules by the excitation signal. This structure can be generated coherent precepts for producing any desirable gait event sequences. Also, in order to reach to any possible phase locking between the oscillators and input signal and as well as exploiting any timing and sequencing of the output gaits, diffusive coupling approach are utilized [19].

Some references like $[16,17]$ used CPG approach with the ability to control and adjusting only the speed of the robot in the duration of the gait transition. In addition, other references like [20,21] proposed the CPG approach with the ability of both adjusting the speed and phase of the foot of the robot in the duration of the gait transition. However, with applying their approach, the ability to define any desirable scenario for gait event sequences is not possible and only the limited set of gaits can be reachable. For reaching to any desirable locomotion scenario, in our previous works such as [22, 23], adaptable CPG networks are developed, which encode foothold positions in respect to the planar obstacles as a forbidden area which can be modulated CPG by feedback signals. Alternatively, references like [24] applied different adaption rules with adaptive oscillators, which called adaptable ring rules. They used their algorithm for reaching to gait switching, while the duty factor and speed would be changed continuously. Moreover, in other references like [25], a CPG model for autonomous decentralized quadruped robot proposed and necessary conditions for the gait transition in CPG network are developed. In this paper, in comparison to another related CPG-based algorithm in the joint space, a CPG network and its modulation are applied for developing motor skills in the Cartesian space. So, the coordinated rhythmic patterns by CPG are used for computing the defined spatio-temporal vectors for footstep planners at different stages. Then this information for each leg is applied to defined Cartesian footstep basis function which generates swing and stance foot location and time adjustment.

Different models of the CPGs are implemented in order to control many types of robots and also varied modes of the locomotion. For instance, the references [26] are implemented a canonical oscillator model on quadruped robot (Aibo), which can generate walking gait, and leakly-integrator neuron model, which allow generation of different gaits with the transition, and reference [27] investigated the conditions of gait transitions for a quadruped robot. Furthermore, a reconfigurable hardware implementation of a CPG-based controller, which is able to generate several gaits for quadruped and hexapod robots, is proposed by [28]. In addition, the reference [29] used CPG for balancing the robot against the external perturbation by coupling CPG with sensory information of the robot. In this paper, all the generated gaits of the CPG are statistically stable, or obviously, COG of the robot is always retained over the polygon which shaped by the supporting feet. For reaching to this feature, a preview controller for computing the ZMP trajectory and COG path for the proposed quadruped robot are utilized [30, 31].

Upon the sensory coupling with the CPG, some references such as $[9,32]$ used input signal from the touch sensors in each leg of the robot, in order to trigger and tune the parameters of the CPG. Also, most of the researchers used these locomotion scenarios in joint space of the robot. Instead, other references [33-36] applied multiple decoupled CPGs with interactions between body dynamics and the environment for interlimb coordination in the joint space of the robot. Conversely, in this paper, foot trajectories are obtained with Cartesian footstep planner by deriving the time gait information and its nominal characteristics from CPG. In this regard, ZMP and COG trajectories are computed and then by using the inverse kinematics relationships for the robot, joint trajectories are calculated for locomotion. This modular and functional organization allow to control the step movements and location of the different joints independently, and at the same time cause to enable programming for different gaits while still keeping the intralimb and interlimb coordination. The structure of the paper is as follows. Initially, physical and experimental set up of the robot is defined and then overall structure of the proposed algorithm is explained briefly. Next, the details sections of the algorithm are discussed, and finally, simulation results are implemented on the robot and compared with other references. 


\section{Material and methods}

\subsection{Material}

\subsubsection{System description}

A small-scale quadruped robot in laboratory condition is used for this research which consists a rectangular plate with dimensions of $20 \times 25 \mathrm{~cm}$ as the main body. The total number of degrees of freedom are eighteen which three actuated degrees of freedom are for each leg and six passive degrees of freedom is for the body, three linear and three rotational degrees of freedoms. The basic kinematic structure of the robot is shown in (Figure 1a).

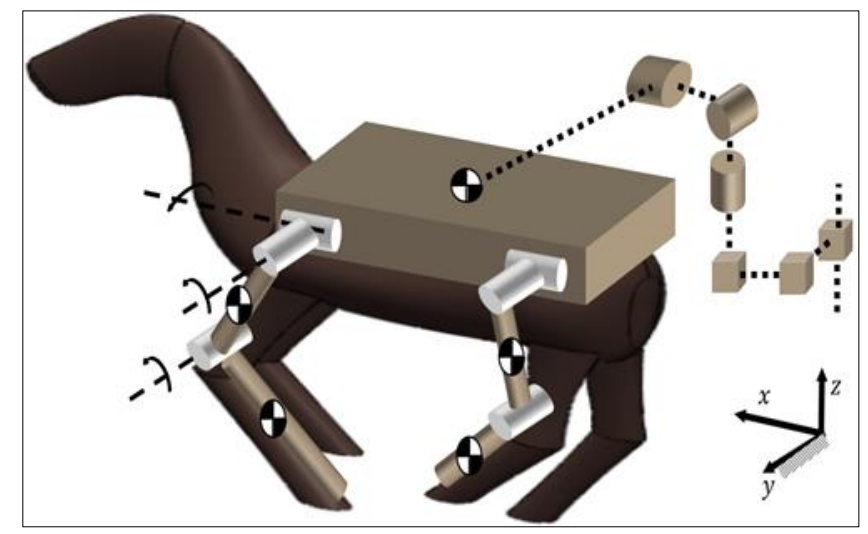

Figure 1 a) Basic kinematic configuration of the robot

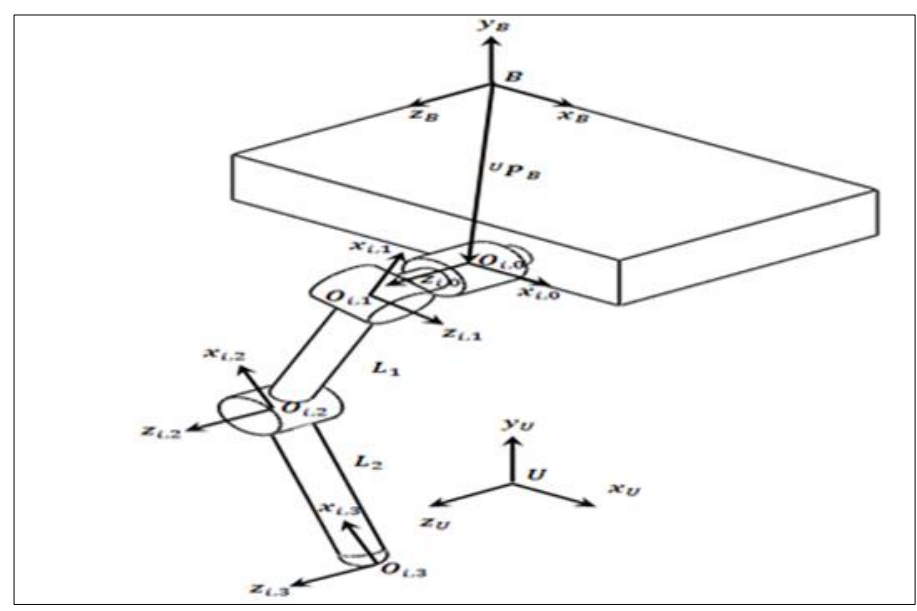

Figure 1 b) Coordination of the body and the $\operatorname{leg}_{-} \mathbf{i}[30]$

Briefly, five coordinates are used for describing motion of the robot, as shown in (Figure 1b), and all of the details about kinematic relationships, validation of model of the robot and so on are expressed deeply in [30, 31]. Each of the four foot of the Quadruped robot, are connected to each corners of the rectangular plate and each of them are equipped with a DC servo motor. The maximum height of the robot is equal to $29 \mathrm{~cm}$, and the robot's body mass is $10 \mathrm{~kg}$ and also the robot is shown in (Figure 1c). Robot actuators are equipped with Dynamixel motors of AX-12 and a potentiometer, which is mounted on the output shaft of the motor as the position feedback, which is measured the rotational position of the motor. The maximum output torque of the motor is $1.62 \mathrm{~N} . \mathrm{m}$ and its voltage is $10 \mathrm{~V}$. In addition, the maximum engine speed is 114 RPM or $11.9 \mathrm{rad} / \mathrm{sec}$ respectively. Furthermore, TTL serial connection is used for sending and receiving signals, and all the calculations are done in MATLAB and then comments will be sent to the motors. Finally, in order to measure the exact position of the body of the robot in the 3D space through image processing an instrument of KINECT is used. It consists of one depth sensor and two Red-Green-Blur (RGB) camera and correspondingly it takes the image of robot with rate of 30 frames per seconds and then analyzed them for computing the COG position. 


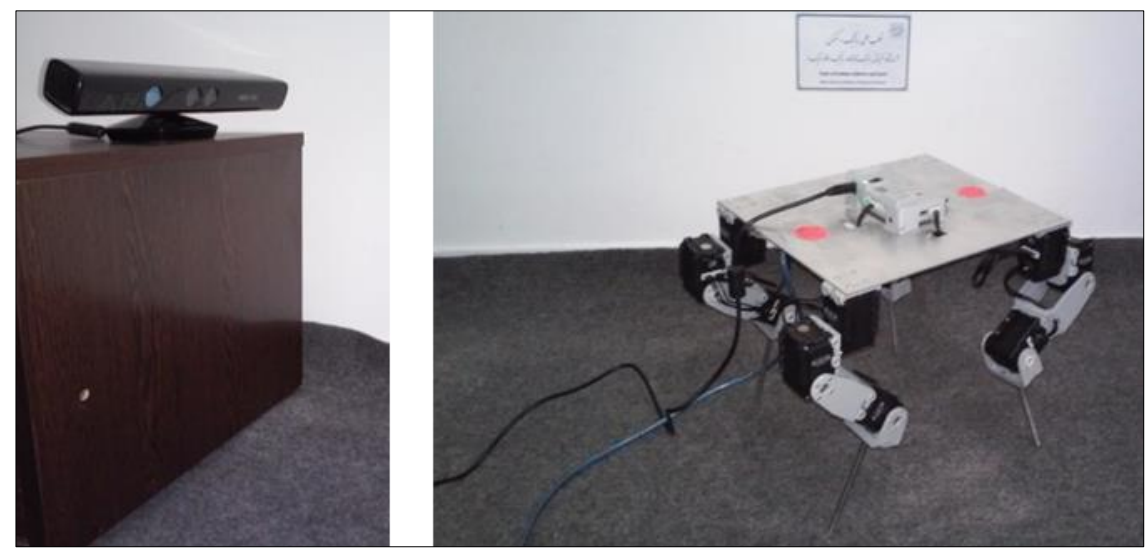

Figure 1 c) Experimental set up of the quadruped robot

\subsection{Method}

\subsubsection{Overall structure of the algorithm}

Primarily, in order to summarize different sections of the proposed algorithm, the schematic block diagram of the algorithm is shown in (Figure 2).

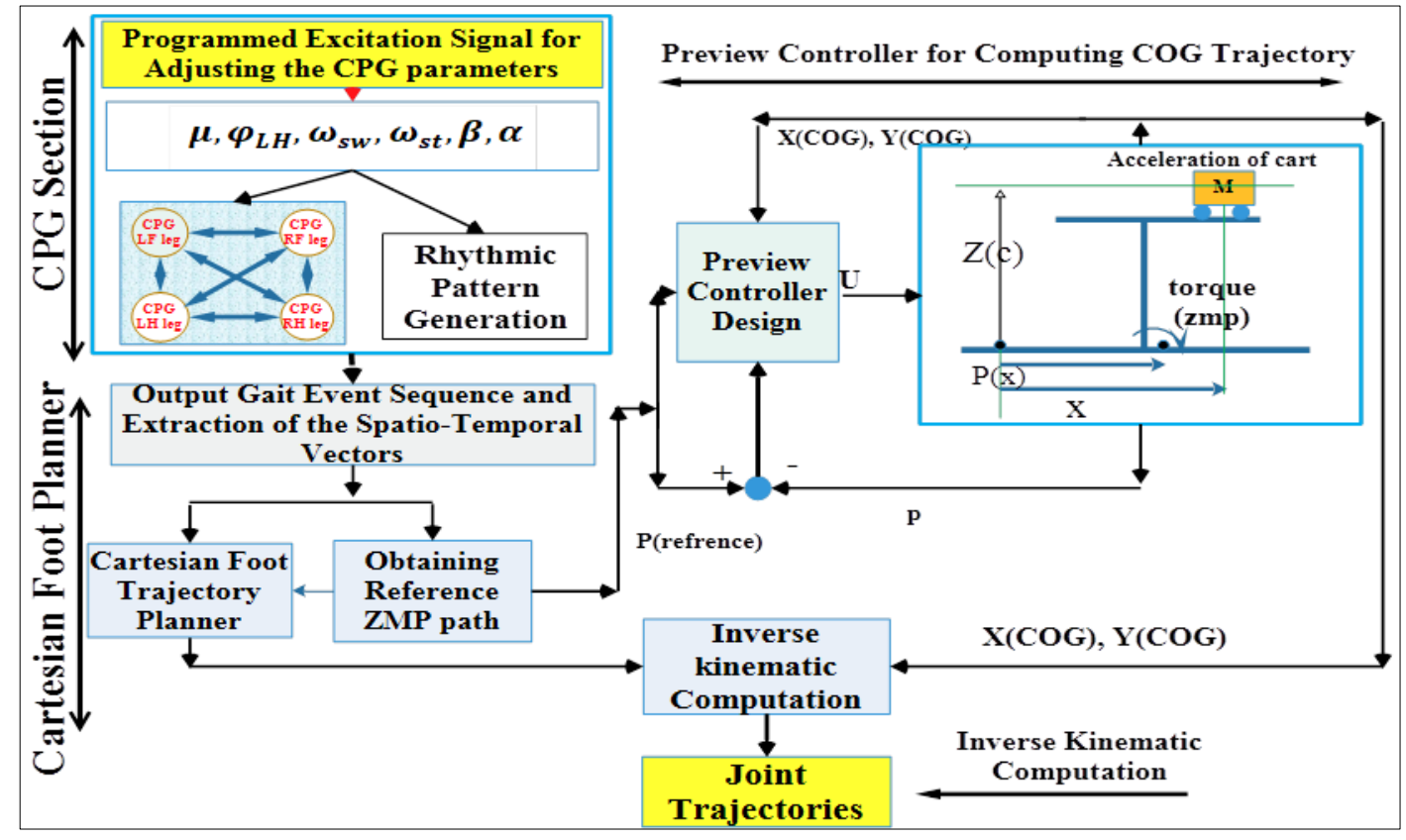

Figure 2 Block diagram of the overall structure of the gait adaption algorithm

Briefly, this algorithm includes of three different sections that are CPG, Cartesian footstep planner and designing Preview controller. In the section of CPG, firstly, the coupling terms between the excitation signal and oscillator's equations are defined. Then, the steps for extracting the gait event sequences, in terms of gait diagram for each foot of the robot, are explained. Subsequently, gait nominal parameters are extracted, from this diagram, in terms of spatiotemporal vectors. Furthermore, trajectories of swing legs along the axis of movement will be calculated through inserting these predefined vectors to defined Cartesian footstep basis function and developing the rules of the related Cartesian footstep planner. Then, the reference ZMP path will be computed on the basis of the footprints of the legs, and consequently, the COG trajectories will be calculated with a preview servo controller. Finally, by having the trajectories of the foot in the Cartesian space and also the COG path, all other joint trajectories are computed through the computations of the inverse kinematics. In this algorithm, robot move in the forward direction and only COG path of the body is needed to determine and all other degrees of freedom are assumed zero. Different sections of the block diagrams are explained in details in further sections. 


\subsubsection{Design of Central Pattern Generator with coupled nonlinear oscillators}

One of the main capability and contribution of the proposed CPG is synthesizing it with novel Cartesian footstep basis function. Primary, CPG parameters network such as amplitude and coupling parameters among the oscillators should be adjusted by programming a drive or excitation signal as some coherent percepts. This structure enables us to adapt and program the gait event sequences, gait nominal values such as step length and duration and interlimb coordination between legs of the robot with defining arbitrary CPG open loop signal. At different steps of the proposed algorithm, these vectors can be put to the Cartesian footstep basis function and consequently the swing foot trajectories in Cartesian workspace along the axis of movements can be planned by Cartesian footstep planner. The brief overall steps of these computations in the CPG section of the algorithm is plotted in (Figure 3).

In this research, selected models for oscillators in the CPG network are chosen based on isochronous oscillator or modified HOPF oscillator. This oscillator has capabilities such as producing both continuous and discrete patterns, low computational cost and smooth pattern modulation in respect to the other oscillators such as Vander poll oscillator. This oscillator has two variables of $\left(P_{X_{i}}, P_{Y_{i}}\right)$ and mathematical equations of the CPG model for i_th oscillator are as follows in equations (1-3) [18]. Also, the approach of wave gait rule was applied in the CPG coupling terms for synchronization in order to create the relative phases relationship between legs of the robot. So, the duty factor and interlimb phase relationship are adjusted continuously and smoothly.

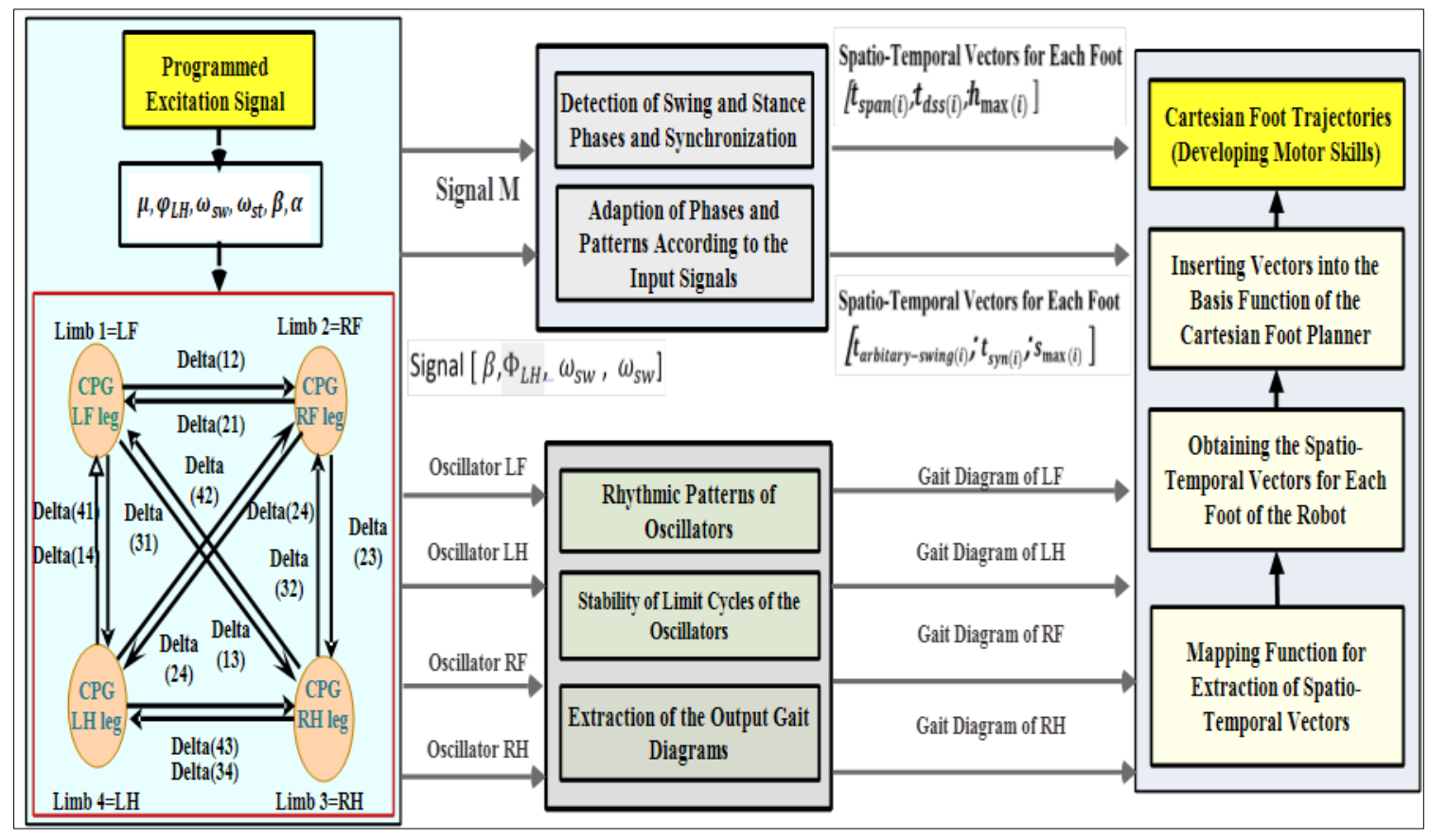

Figure 3 Flow chart of the desirable features and spatio-Temporal vectors extraction from the CPG model for computing the Cartesian footstep trajectories

$$
\begin{gathered}
{\left[\begin{array}{c}
\dot{P}_{x_{i}} \\
\dot{P}_{y_{i}}
\end{array}\right]=\left[\begin{array}{cc}
\alpha\left(\mu_{i}-r_{i}^{2}\right) & -\omega_{i} \\
\omega_{i} & \alpha\left(\mu_{i}-r_{i}^{2}\right)
\end{array}\right]\left[\begin{array}{l}
p_{x_{i}} \\
p_{y_{i}}
\end{array}\right](1)} \\
\omega_{i}=\frac{\omega_{\text {stance-duration }}}{1+e^{b y_{i}}}+\frac{\omega_{\text {swing-duration }}}{1+e^{-b p_{y_{i}}}}(2) \\
\omega_{\text {stance-duration }}=\frac{1-s_{\text {ratio }}}{s_{\text {ratio }}} \omega_{\text {swing-duration }}
\end{gathered}
$$

In these equations, $\mathrm{r}$ is defined as $\mathrm{r}=\sqrt{x^{2}+y^{2}}$ and amplitude of oscillations is adjusted by parameters of $A=\sqrt{\mu}$. Also, the oscillation frequency is determined by $\omega_{i}$ for each oscillator, and it varies between two different frequencies. So, this enables us to adjust the swing and the stance phase independently, and accordingly the duty factor of the robot. 
Also, $p_{x_{i}}$ and $p_{y_{i}}$ are i_th oscillator outputs, and the parameter $\mathrm{b}$ is responsible for adjusting the switching rate between the swing and stance phases. Finally, $\alpha$ adjusts the convergence speed of the limit cycle of the oscillators. Also, coupling structure between the oscillators of the CPG network is shown in (Figure 3). In summary, in this network, delta (ij) represents the interaction or coupling terms between the oscillators, (i, j) ? $\{\mathrm{LF}, \mathrm{RF}, \mathrm{LH}, \mathrm{RH}\}$. For instance, the term $\mathrm{LF}$ means left and front of the foot of the robot, and also each node of the network is one oscillator which at last encodes gait diagram for one foot of the robot. In this network, coupling approach between the oscillators must be such a way in which any particular phase differences between the oscillators can be obtained. For reaching to this condition, the rotation matrix under the symbols of $\mathrm{R}\left(\theta_{i j}\right)$ is used in the CPG equations, which the term of $\theta_{i j}$ indicates the relative phase difference between the oscillator $\mathrm{i}$ and $\mathrm{j}$ respectively. It controls and adjusts the periodic stepping sequence, and also the diffusive coupling is used in the CPG. These coupling equations are presented in the below equations [22, 23].

$$
\begin{gathered}
{\left[\begin{array}{c}
\dot{P}_{x_{i}} \\
\dot{P}_{y_{i}}
\end{array}\right]=\left[\begin{array}{cc}
\alpha\left(\mu_{i}-r_{i}^{2}\right) & -\omega_{i} \\
\omega_{i} & \alpha\left(\mu_{i}-r_{i}^{2}\right)
\end{array}\right]\left[\begin{array}{l}
p_{x_{i}} \\
p_{y_{i}}
\end{array}\right]+\sum_{j \neq i} R\left(\theta_{i j}\right)\left[\begin{array}{l}
p_{x_{i}} \\
p_{y_{i}}
\end{array}\right](4 \mathrm{a})} \\
\mathrm{R}\left(\theta_{i j}\right)=\left[\begin{array}{cc}
\cos \left(\theta_{i j}\right) & -\sin \left(\theta_{i j}\right) \\
\sin \left(\theta_{i j}\right) & \cos \left(\theta_{i j}\right)
\end{array}\right] \mathrm{R}\left(\theta_{i j}\right)=R^{-1}\left(\theta_{i j}\right)(4 \mathrm{~b}) \\
\dot{P}_{x_{i}}=f_{x, 1}\left(p_{x 1}, p_{y 1}\right)+\mathrm{K}\left(\left[\cos \left(\theta_{i j}\right)-\sin \left(\theta_{i j}\right)\right] \cdot\left[\begin{array}{l}
p_{x_{i}} \\
p_{y_{i}}
\end{array}\right]\right)(4 \mathrm{c}) \\
\dot{P}_{y_{i}}=f_{y, 1}\left(p_{x 1}, p_{y 1}\right)+\mathrm{K}\left(\left[\sin \left(\theta_{i j}\right) \cos \left(\theta_{i j}\right)\right] \cdot\left[\begin{array}{l}
p_{x_{i}} \\
p_{y_{i}}
\end{array}\right]\right)(4 \mathrm{~d})
\end{gathered}
$$

In above equations, the coupling parameter of $\mathrm{K}$ determines the convergence time. Principally, quadruped gaits are characterized with duration of their stance phases, relative phases, and duty factor values. In this research, for exploiting the beneficial advantages of wave gait rule, like smooth behavior for transition and its stability, these rules are applied for gait planning. According to the wave gait rule for synchronization, relative phases relationship between foots of the robot is followed from the duty factor parameter as in below in Equation (5a) and Equation (5b).

$$
\begin{gathered}
\phi_{L F}=0 \cdot \phi_{R F}=0.5 \cdot \phi_{R H}=\phi_{L H}-0.5(5 \mathrm{a}) \\
\Phi_{L F}=0, \Phi_{R F}=0.5, \Phi_{L H}=\beta, \Phi_{R H}=\beta-0.5(5 \mathrm{~b})
\end{gathered}
$$

In Equation (5a), $\phi_{L H}$ is defined as phase gait and $\beta$ is the duty factor. By substituting these relationships to Equation (6), relative phases of oscillators in terms of phase gait can be calculated and obtained as in Equation (7).

$$
\theta_{i j}=\left(\phi_{i(\text { oscilator })}-\phi_{j(\text { oscilator })}\right) 2 \pi(6)
$$

$\theta_{21}=-\pi, \theta_{31}=-\phi_{L H}, \theta_{41}=-\pi-\phi_{L H}, \theta_{12}=\pi$,

$$
\begin{gathered}
\theta_{32}=\pi-\phi_{L H}, \theta_{42}=-\phi_{L H}, \theta_{13}=\phi_{L H} \\
\theta_{23}=-\pi+\phi_{L H}, \theta_{43}=-\pi, \theta_{14}=\pi+\phi_{L H}, \\
\theta_{24}=\phi_{L H}, \theta_{34}=\pi(7)
\end{gathered}
$$

As a consequence, a network of oscillators with ability of controlling the phase coupling or their synchronized patterns between oscillators can be obtained. Then, by re-arranging according to these coupling for relative phases, and in the terms of phase gait or $\phi_{L H}$, the state space equations for the oscillator LF becomes as follows in Equation (8). Also, with substitution of variables for oscillator LF, the extended format cab be written as Equation (9). Others equations for the oscillators can be computed in this way. 


$$
\begin{gathered}
\dot{P}_{x_{1}}=f_{x, 1}\left(p_{x 1}, p_{y 1}\right)+c p_{x}(2)-s p_{y}(2)+c p_{x}(3)-s p_{y}(3)+c p_{x}(4)-s p_{y}(4) \\
\dot{P}_{y_{1}}=f_{y, 1}\left(p_{x 1}, p_{y 1}\right)+s p_{x}(2)+c p_{y}(2)+s p_{x}(3)+c p_{y}(3)+s p_{x}(4)+c p_{y}(4)(8) \\
\dot{P}_{x_{1}}=f_{x, 1}\left(p_{x 1}, p_{y 1}\right)+\mathrm{k} \times\left[\left(p_{x}(2) \times \cos (-\pi)-p_{y}(2) \times \sin (-\pi)+p_{x}(3) \times \cos \left(-\phi_{L H}\right)-\right.\right. \\
\left.\left.p_{y}(3) \times \sin \left(-\phi_{L H}\right)+p_{x}(4) \times \cos \left(-\pi-\phi_{L H}\right)-p_{y}(4) \times \cos \left(-\pi-\phi_{L H}\right)\right)\right] \\
\dot{P}_{y_{1}}=f_{y, 1}\left(p_{x 1}, p_{y 1}\right)+\mathrm{K} \times\left[\left(p_{x}(2) \times \sin (-\pi)+p_{y}(2) \times \cos (-\pi)+p_{x}(3) \times \sin \left(-\phi_{L H}\right)+\right.\right. \\
\left.\left.p_{y}(3) \times \cos \left(-\phi_{L H}\right)+p_{x}(4) \times \sin \left(-\pi-\phi_{L H}\right)+p_{y}(4) \times \cos \left(-\pi-\phi_{L H}\right)\right)\right](9)
\end{gathered}
$$

For existing the ability to adjust duty factor, phase gait of the CPG network and others features coherently, a programmed excitation signal with term of $\mathrm{M}$ is used. Therefore, it can initiate gaits, switch among gaits and has ability to define local phase synchronization between patterns. This signal strength is planned onto the different sets of the CPG parameters, and therefore result in the different gait planning scenarios. For existing the functional relationship between drive signal and others parameters of CPG, such as duty factor and phase gait, a saturated value between signals $\mathrm{M}$, value of excitation signal, and each parameter in the network can be defined. Then, below or above of this value, the linear function with an arbitrary rate of increase or decrease for the desired parameter can be chosen. So, the generated patterns are a combination of intrinsic dynamics of the CPG and input signal which can be utilized in order to design diverse timing and spanning the gait parameters. Also, by existing functional relationship between the excitation signal and parameters in CPG, adaptable patterns are generated. In this research, relationship functions between the excitation signal and duty factor, phase gait of the CPG network and others are linear in duration of the time of the simulation. Moreover, for detection and defining of swing or stance phases in oscillators patterns, when $p_{y_{i}}<0$, the leg is in the stance phase, and when $p_{y_{i}}>0$, the leg is in swing phase. In this paper, at each step all of the CPG equations are computed and numerically integrated using the Runge-Kutta with a variable time step of $1 \mathrm{~ms}$.

\subsubsection{Cartesian footstep planner}

A primary step in the design of a gait is the determination of the related workspace of the robot and then defining the sequence of foot lifting in Cartesian space. By using the forward kinematic equations and specification of the robot, our robot's workspace is determined and plotted in (Figure 4a). This suggestion ensures us that step length limitation can be specified with respect to the workspace. In this research, the sequence of 1423 is chosen for locomotion, which has maximum stability and as well as it is same as the locomotion of the animals. Also, other possible sequences for the robot is plotted in (Figure 4b) respectively.

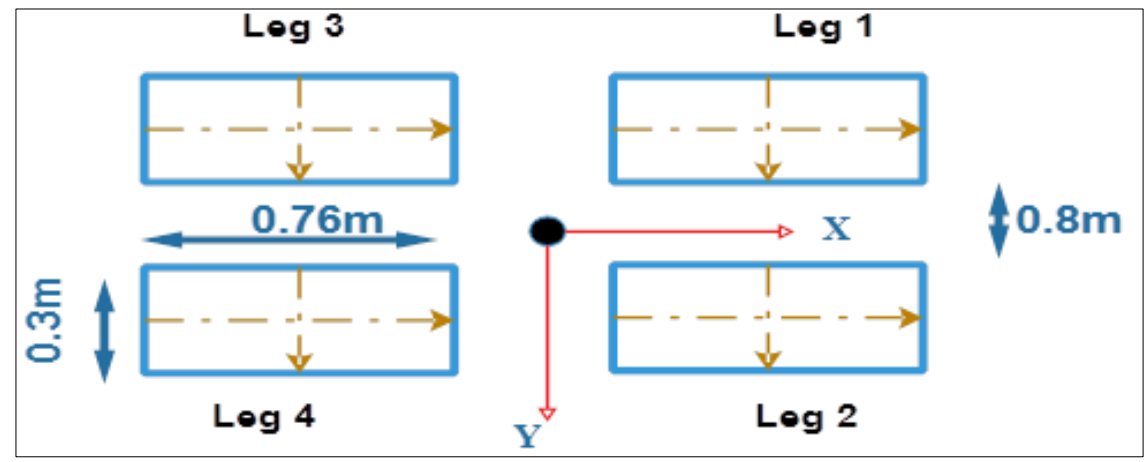

Figure 4 a) Workspace of the robot 


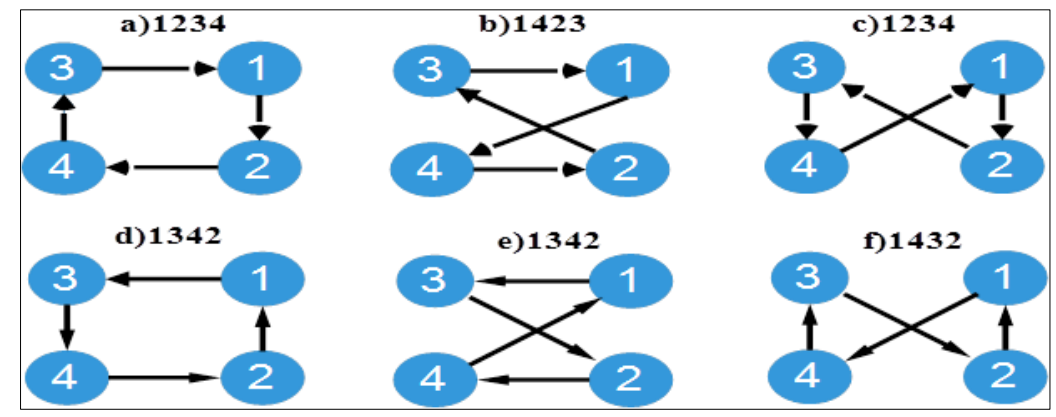

Figure 4 b) All possible sequences of leg lifting of the robot

Footstep trajectories in Cartesian space will be planned as a function of the output gait diagram or synchronized patterns of the CPG at two distinct steps or stages. Also, in this methodology, adjustment of the walking trajectory can be programmed properly due to the inherent favorable dynamic properties of the CPGs. Primarily, in the simulations, depending to the strength values of the excitation signal, different set for the CPG parameters can be computed, which can be regarded to different gait event sequence or gait diagram. For instance, these time scale information, swing and stance time and variation of swing and stance times, in one gait diagram are defined in (Figure 5a). Also, at the first stage of the algorithm, these nominal gait characteristics can be saved as different spatio-temporal vectors for each foot. Next, they can be applied in the second stage of the algorithm to Cartesian footstep basis function for deriving swing and stance location and timing. Typical definition of these parameters in the Cartesian space is plotted in (Figure 5b) respectively. Furthermore, in the second stage, each of these spatio-temporal vectors, which contains time gait information, can be attained from the gait diagram. Next, they are imported to the Cartesian footstep basis function which is designed for the Cartesian footstep planner.

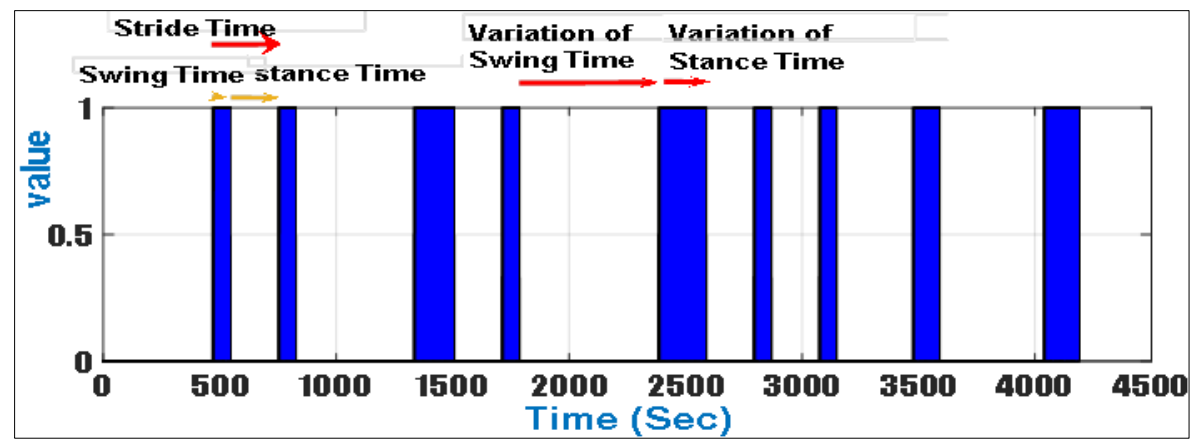

Figure 5 a) Defining time gait information in one output gait diagram of the CPG

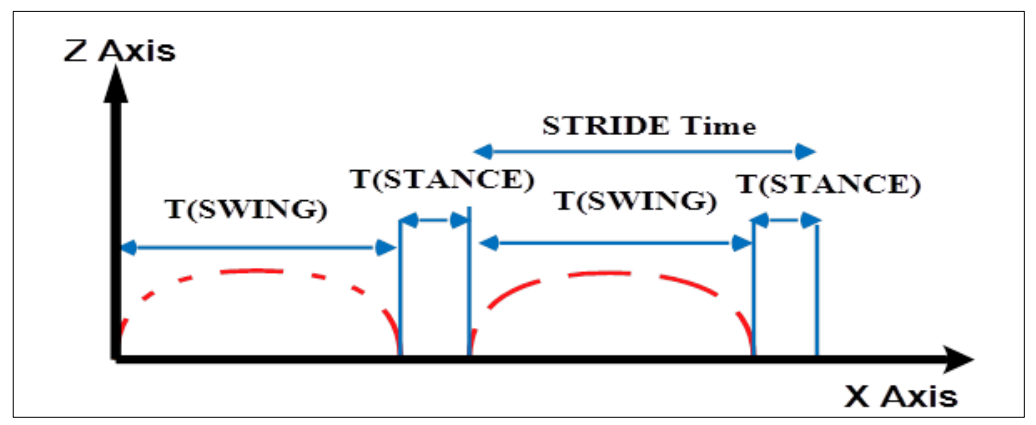

Figure 5 b) General definitions of the axis of movement and parameters

In this section, firstly, the equations of the Cartesian footstep basis function are developed and then necessary spatiotemporal vectors for inserting to these equations are defined and developed respectively. In Cartesian space $\mathrm{x}$ and accordingly $p_{x_{\text {foot }(i)}}$ axis is in the forward direction of robot and $\mathrm{y}$ axis is in the direction of width of robot, and finally, $\mathrm{z}$ axis or $p_{z_{\text {foot }(i)}}$ is in the direction of height of the robot. The key basis function for generating swing foot trajectory for each leg in Cartesian space are chosen as below equations. Also, the y-components of the footprints are similar. 


$$
\begin{gathered}
\ddot{p}_{x_{\text {foot }(i)}}=c_{1} \sin \left(\frac{2 \pi}{T_{S}} t\right)(10) \\
\ddot{p}_{z_{\text {foot }(i)}}=\left\{\begin{array}{c}
c_{4} \sin \left(\frac{4 \pi}{t_{s}} t\right) t \leq \frac{t_{s}}{2} \\
c_{7} \sin \left(4 \pi\left(\frac{t}{t_{s}}-\frac{1}{2}\right)\right) t \geq \frac{t_{s}}{2}
\end{array}\right.
\end{gathered}
$$

Where trajectories coefficients are $c_{1}, c_{4}$ and $c_{7}$, which should be determined from the predefined initial and final conditions. Basically, the key reason for choosing these basis function are its features, such as smooth behavior pattern for tip of the foot of the robot in Cartesian space and as well as for velocity and acceleration at initial and final period of each stride. Moreover, the speed and acceleration of swing legs at the time corresponding to the maximum height of the foot and also initial and final velocities of swing legs are set equivalent to zero. Constant terms in above equations can be obtained by applying double integration for both $P_{x, \text { foot }(i)}$ and $P_{z, f o o t(i)}$ directions, which are derived and computed as Equations (12-15).

$$
\begin{gathered}
\dot{p}_{x_{\text {foot }(i)}}=-c_{1}\left(\frac{t_{s}}{2 \pi}\right) \cos \left(\frac{2 \pi}{t_{s}} t\right)+c_{2}(12) \\
p_{x_{\text {foot }(i)}}=-c_{1}\left(\frac{t_{s}}{2 \pi}\right)^{2} \sin \left(\frac{2 \pi}{t_{s}}\right)+c_{2} t+c_{3}(13) \\
\dot{p}_{z_{\text {foot }(i)}}=\left\{\begin{array}{c}
-c_{4}\left(\frac{t_{s}}{4 \pi}\right) \cos \left(\frac{4 \pi}{t_{s}} t\right)+c_{5} t \leq \frac{t_{s}}{2} \\
-c_{7}\left(\frac{t_{s}}{4 \pi}\right) \cos \left(4 \pi\left(\frac{t}{t_{s}}-\frac{1}{2}\right)\right)+c_{8} t \geq \frac{t_{s}}{2}
\end{array}\right. \\
p_{z_{\text {foot }(i)}}=\left\{\begin{array}{c}
-c_{4}\left(\frac{t_{s}}{4 \pi}\right)^{2} \sin \left(\frac{4 \pi}{t_{s}}\right)+c_{5} t+c_{6} t \leq \frac{t_{s}}{2} \\
-c_{7}\left(\frac{t_{s}}{4 \pi}\right)^{2} \sin \left(4 \pi\left(\frac{t}{t_{s}}-\frac{1}{2}\right)\right)+c_{8} t+c_{9} t \geq \frac{t_{s}}{2}
\end{array}\right.
\end{gathered}
$$

Then, by applying the defined conditions, the final relationships for basis function are computed as below:

$$
\begin{gathered}
p_{x_{\text {foot }(i)}}=s_{\max }\left(\frac{t}{t_{s}}-\frac{1}{2 \pi} \sin \left(\frac{2 \pi}{t_{s}} t\right)(16)\right. \\
p_{z_{\text {foot }(i)}}=\left\{\begin{array}{c}
2 h_{\max }\left(\frac{t-t 0}{t_{s}}-\frac{1}{4 \pi} \sin \left(\frac{4 \pi}{t_{s}} t-t 0\right) t \leq \frac{t_{s}}{2}\right. \\
2 h_{\max }\left(1-\frac{t-t o}{t_{s}}+\frac{1}{4 \pi} \sin \left(4 \pi\left(\frac{t-t 0}{t_{s}}-\frac{1}{2}\right)\right) t \geq \frac{t_{s}}{2}\right.
\end{array}\right. \\
p_{x_{\text {foot }(i)}}=s_{\max }\left(\frac{t-t_{0}}{t_{s}}\right)-\frac{1}{2 \pi} \sin \left(\frac{2 \pi}{t_{s}}(t-t o)\right)(18)
\end{gathered}
$$

As illustrated in (Figure 5a), each sequential duration of swing and stance periods can be extracted from each gait diagram independently. As a result, different spatio-temporal vectors which is necessary for drawing the trajectories of swing legs along the axis of movement, are defined and computed. The final form of Cartesian footstep basis function, with substitution of the defined spatial vectors, are represented by below equations in respect to the $\mathrm{z}$ and $\mathrm{x}$ axis. 


$$
\begin{aligned}
& \int 2 h_{\max (i)}\left(\frac{t_{s p a n(i)}}{t_{d s s(i)}}-\frac{1}{4 \pi} \sin \left(\frac{4 \pi}{t_{d s s(i)}} * t_{\operatorname{span}(i)}\right)\right. \\
& p_{z_{\text {foot }(i)}}=\left\{\begin{array}{c}
\text { if } t_{\text {span }(i)} \leq \frac{t_{d s s(i)}}{2} \\
2 h_{\max (i)}\left(1-\frac{t_{s p a n(i)}}{t_{d s s(i)}}+\frac{1}{4 \pi} \sin \left(4 \pi\left(\frac{t_{s p a n(i)}}{t_{d s s(i)}}-\frac{1}{2}\right)\right)\right.
\end{array}\right. \\
& \text { if } t_{\text {span }(i)} \geq \frac{t_{d s s(i)}}{2} \\
& p_{x_{\text {foot }(i)}}=s_{\max (i)}\left(\frac{t_{\text {span }(i)}+t_{\text {stance }(i)}}{t_{\text {swing }(i)}}\right)-\frac{1}{2 \pi} \sin \left(\frac{2 \pi}{t_{\text {swing }(i)}}\left(t_{\text {span }(i)}+t_{\text {stance }(i)}\right)\right)
\end{aligned}
$$

In the proposed algorithm, the vectors of $t_{\text {span(i) }}$ is a one dimensional vector which its components are sequential duration of swing and stance periods of the gait diagram, which begins from time steps of the simulation. In (19) and (20), $\mathrm{h}_{\max }$ is the maximum height of the tip of swing leg or a step height, and $\mathrm{s}_{\max }$ is the step length and $t_{\text {arbitary-swing(i) }}$ is the arbitrary swing time in the Cartesian space. These values can be defined according to the physical characteristics of the robot. So, $t_{\text {arbitary-swing(i) }}, h_{\max (i)}$ and $s_{\max (i)}$, can be given constant values in duration of simulation or they can be varied arbitrary. In this paper, these vectors have constant values in the duration of the simulations. Also, the defined vectors of $t_{\text {duratioon,;swing,stance(dss)(i) }}$ is the vectors that its components are created by inserting the swing and stance periods of the gait event sequences separately and iteratively. Moreover, the even components of the defined vector of $t_{\text {syn(i) }}$ can be completed by computation of the stance phases duration from the gait diagram for each leg independently, and next, accumulating these values sequentially and then inserting to next components of the vector successively with the negative sign. Next, the secondly odd components of this vector are created by the sequential duration of the swing

\begin{tabular}{|c|c|}
\hline $\begin{array}{l}\text { Spatio-temporal } \\
\text { Vectors }\end{array}$ & $\begin{array}{c}\text { Extraction the Components of Vectors from the Gait } \\
\text { Diagram }\end{array}$ \\
\hline$t_{\text {span(i) }}$ & $\begin{array}{l}{[0,3.28,3.98,6.06, \quad 6.76,9.84,11.54,15.64,16.34,19.72,21.72,} \\
26.42,27.12,29.2,29.9,32.38,33.48,37.18,38.68,41.68]\end{array}$ \\
\hline $\begin{array}{l}\mathrm{t} \\
\text { swing,stance(dss)(i) }\end{array}$ & $\begin{array}{l}{[3.28,0.7,2.08,0.7,3.08,1.7,4.1,0.7,3.38,2,4.7,0.7,2.08,0.7,2.48,1.1} \\
\text {,3.7,1.5,3] }\end{array}$ \\
\hline $\mathrm{t}_{\text {arbitary-swing(i) }}$ & {$[0.7]$} \\
\hline $\mathrm{h}_{\max (\mathrm{i})}$ & {$[0.1]$} \\
\hline $\mathrm{S}_{\max (\mathrm{i})}$ & {$[0.76]$} \\
\hline $\mathrm{t}_{\text {syn(i) }}$ & $\begin{array}{l}{[0,-3.28, \quad 3.98,-5.36, \quad 6.76,-8.44, \quad 11.54,-12.54, \quad 16.34,-15.92,} \\
21.72,-20.62,27.12,-22.7,29.9,-25.18,33.48,-28.88,38.68]\end{array}$ \\
\hline
\end{tabular}
phases of the gait diagram respectively. These spatio-temporal vectors for the sample gait diagram of the (Figure 5a) computed and written as below in (Table 1).

Table 1 Defining the spatial vectors components for gait diagram of (Figure 5a)

\subsubsection{Deriving COG path generation from Cartesian footstep planner}

In this section of the algorithm, the appropriate path for the COG of the robot should be designed in a way in which any appropriate gait by the CPG open loop signal command can be planned for the robot locomotion in the workspace by ensuring the stability of the robot. In this paper, ZMP criterion in order to retain the stability of robot during locomotion is developed. In this criterion, when three feet of the robot like (leg LH, RF and RH) are on the ground, the projection of the image of the center of mass of the robot should be positioned on the triangular area where the vertices of the three legs are on the ground. Consequently, following this approach guarantees zero moments of the external forces and also prohibits any tumbling moments. For deriving the ZMP equations, since the weight of all the legs of the robot is ignorable in relative to the mass of the main body of the robot, the angular moment effects of all legs can be ignored. So, a simple model or point mass model at the COG of the main body can be calculated for the quadruped robot. By using the 
developed method and assumptions which was proposed by [34, 35], the ZMP equations along $\mathrm{x}$ and $\mathrm{z}$ axes can be modeled and represented as:

$$
\begin{aligned}
& P_{Z M P}^{x}=P_{G}^{x}-\omega \ddot{P}_{G}^{x}(21) \\
& P_{Z M P}^{Z}=P_{G}^{Z}-\omega \ddot{P}_{G}^{Z}(22)
\end{aligned}
$$

Where, $P_{Z M P}^{x}, P_{Z M P}^{Z}$ is the position of ZMP along the $\mathrm{x}$ and $\mathrm{z}$ axes, $P_{G}^{x}, P_{G}^{Z}$ is the positions of COG along the $\mathrm{x}$ and $\mathrm{z}$ axes, $\ddot{P}_{G}^{x}$, $\ddot{P}_{G}^{z}$ is the COG acceleration along the $\mathrm{x}$ and $\mathrm{z}$ axes and finally, the $\omega$ is assumed the constant value [34, 35]. For converting the predefined ZMP equations to appropriate dynamical systems, we specified $u_{x}$ as the time derivative of the COG acceleration and then ZMP equations can be developed as appropriate discrete state space models as below [37, 38]:

$$
\begin{gathered}
\frac{d}{d t} \ddot{x}=u_{x} ; \frac{d}{d t}\left[\begin{array}{l}
x \\
\dot{x} \\
\ddot{x}
\end{array}\right]=\left[\begin{array}{lll}
0 & 1 & 0 \\
0 & 0 & 1 \\
0 & 0 & 0
\end{array}\right]\left[\begin{array}{l}
x \\
\dot{x} \\
\ddot{x}
\end{array}\right]+\left[\begin{array}{l}
0 \\
0 \\
1
\end{array}\right] u_{x} \\
\dot{P}_{x}=[10-\omega]\left[\begin{array}{l}
x \\
\dot{x} \\
\ddot{x}
\end{array}\right] \\
X(k+1)=A X(k)+B u(k) ; P(k)=C X(k) \\
\text { Where X }(k)=[X(K T) \dot{X}(K T) \ddot{X}(K T)] T \\
U(K)=U_{x}(K T) ; P(K)=P_{X}(K T) \\
A_{\text {CONTROLLER }}=\left[\begin{array}{ccc}
1 & T & \frac{T^{2}}{2} \\
0 & 1 & T \\
0 & 0 & 1
\end{array}\right] ; B_{\text {CONTROLLER }}=\left[\begin{array}{l}
\frac{T^{3}}{6} \\
\frac{T^{2}}{2} \\
T
\end{array}\right] ; C=[10-\omega](23)
\end{gathered}
$$

Where $\mathrm{T}$ is the sampling time. According to the above modelling and ZMP equations, when the COG acceleration becomes zero, the COG path will be similar to designing the ZMP reference path.

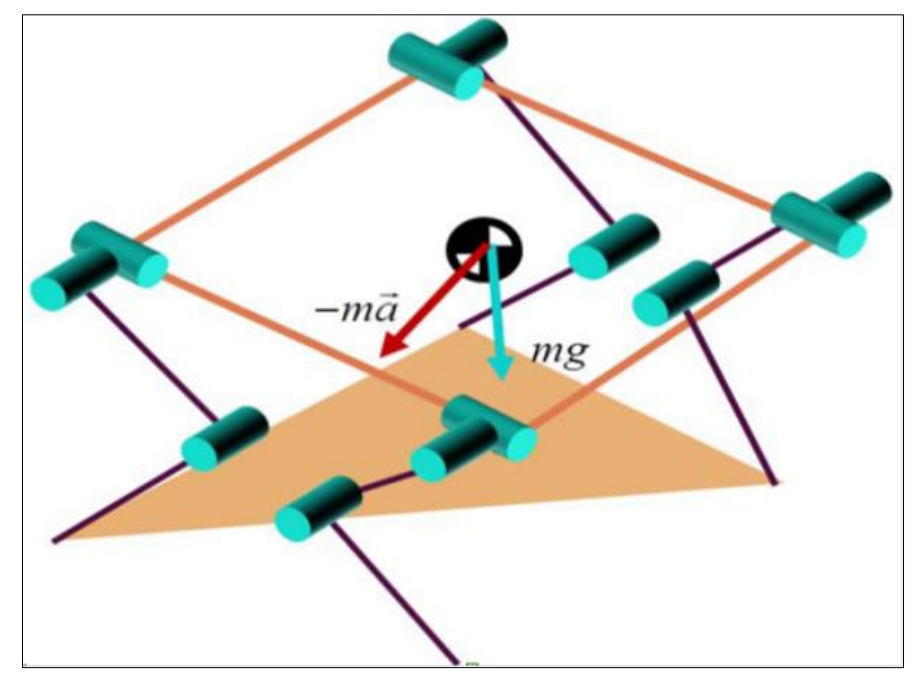

Figure 6 ZMP criterion for maintaining the stability of the robot in different gaits [31] 
As a result, ZMP reference path along the $\mathrm{x}$ and $\mathrm{y}$ axes can be calculated based on the accurate location of the footprints and defining the support polygon triangle of stance foots on the ground plane. In briefly, by mapping the total extracted gait parameters to Cartesian footstep planar, the location of footprints for any gait scenario is predetermined and this condition for the quadruped robot is shown in the (Figure 6). So, when the robot moves, the center of mass of the robot should always be positioned in the related triangular which is obtained with footholds in the duration of locomotion. Next, the reference ZMP trajectory is calculated in the duration of the motion and a reference ZMP trajectory based on the support polygon triangle of stance foots is obtained and then desired COG path generation is calculated with preview controller. So, the COG path generation problem can be modeled as a tracking problem.

After discretization process of the ZMP equations in (23), a ZMP tracking servo controller is designed for compensation of ZMP tracking error and additional constraints associated from terminal ZMP and initial and final conditions [22-23]. Based on the reference ZMP trajectory, $p^{r e f}(\mathrm{i})$, the control problem can be determined as:

$$
J_{\text {CONTROL }}=\sum_{I=K}^{\delta}\left\{Q_{e} e(i)^{2}+\Delta x^{T}(i) Q_{x} \Delta x(i)+R \Delta u^{2}(i)\right\}
$$

Subject to ZMP equations (equations 23)

$$
\text { Initial and final conditions: } X_{1}=X_{0}, Y_{N}=y_{f}=C_{f} X_{f}
$$

Where, $\mathrm{e}(\mathrm{i})=\mathrm{p}(\mathrm{i})-p^{\text {ref }}(\mathrm{i})$ is the error between the measured and desired ZMP, and $Q_{e}, R>0, Q_{x}$ is $3 \times 3$ symmetric nonnegative matrix and $y_{f}$ is the terminal desired ZMP. Next, for minimizing of this performance index for optimal preview controller, input controller which can minimize this index is calculated as follows.

$$
U_{c o n}=-G_{i} \sum_{i=0}^{k} e(k)-G_{x} x(k)-\sum_{j=1}^{N_{L}} G_{P}(j) p^{r e f}(k+j)
$$

Where $G_{i}, G_{x}$ and $G_{P}(j)$ are the gained calculated from the $Q_{e}, Q_{x}, \mathrm{R}$ and parameters of (23) [36]. Also, since the final conditions are only defined, these equations must be resolved backwards. When the $Q_{e}, Q_{x}, \mathrm{R}$ matrices are obtained, the optimal input, $U_{\text {con }}$, can be computed by equation (25) and the COG path can be computed by equation (23). In summary, the COG path generation algorithm is plotted in (figure 7).

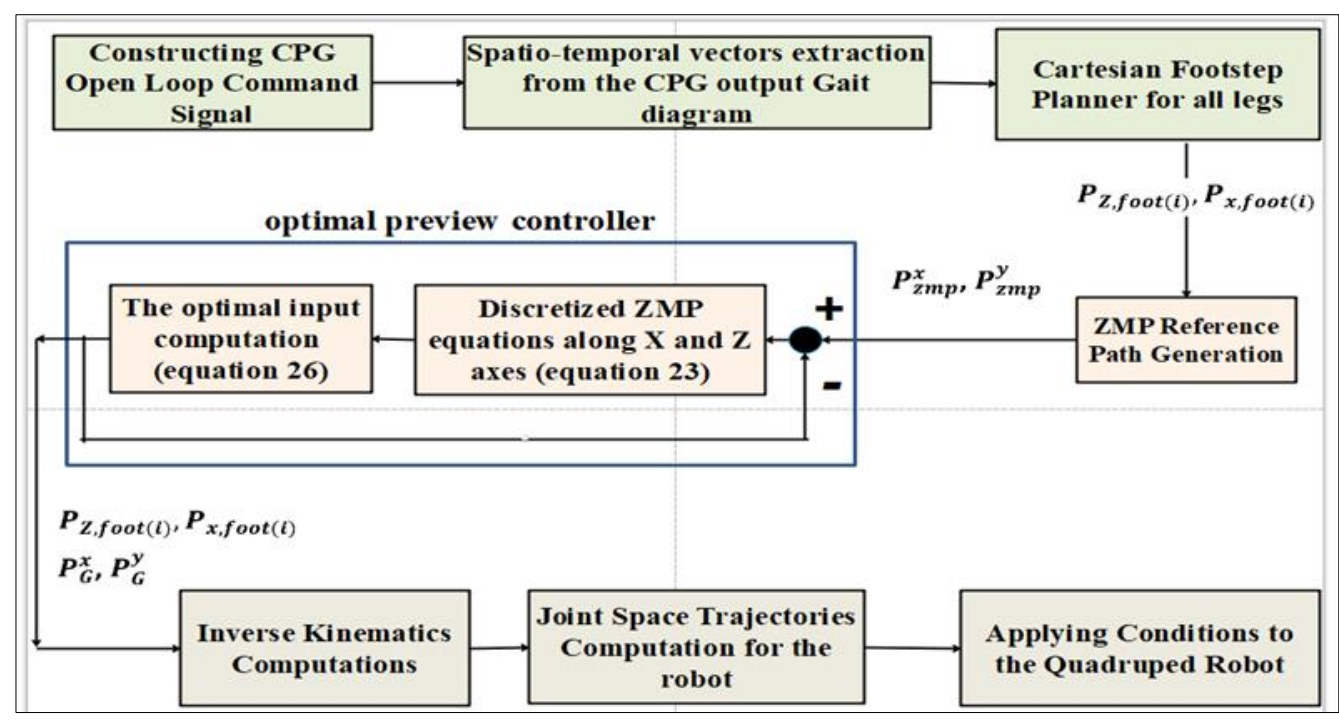

Figure 7 Block diagram for tracking the ZMP reference and obtaining the COG path

In summary, when the desired ZMP trajectory is created with following of support polygon region of stance foots according to designed footprints plan for $N_{L}$ future step at every sample time, the optimal controller minimizes above performance index or tracking error and accordingly its input can be gained. Next, the joint angle trajectories for all legs for moving can be found by the COG path and the Cartesian footstep planner by using inverse Kinematics computation. Finally, the proposed algorithm can be implemented on a quadruped robot by using a feed-forward control and the attained experimental results will be discussed in the following sections. 


\section{Results}

Principally, the excitation signal can be programmed in many ways depending to the desired and favorable Cartesian gait planning. In this section for creating Cartesian motor skills for the robot, the excitation or modulation signal, $\mathrm{M}$, is functioned to change linearly between different designed values in duration of the simulation, and consequently parameters of the CPG are adapted linearly. In other exact words, by defining the different priori information or strength values for the modulation signal, different set for the CPG parameters which are regarded to different possible gait event sequence, can be induced. One of the possible locomotion scenarios, which is functioned between modulation signal and set of the CPG parameters, is commended and shown in (Figure 8). On the other hand, for sending the coherent percept for inducing the gait event sequence, different threshold values, or strength value, are utilized for modulation signal. For instance, the value of $\mathrm{M}=1$ and below of it, is correspond to the walking gait, and when the value of $M$ is changed from 1 to 2.5, the gait transition from walking to trot gait is happened or vice versa. Moreover, an arbitrary transition time can be functioned in order to choice and switch between different principal gaits of the quadruped robot, according to the limitation setup of the robot or the desired Cartesian behavioral context.

In this scenario, the gait event sequence of the robot is changed from walking to trot gait by the time of 17 seconds and allocating 3.5 seconds for gait transition. Then, suddenly its gait is changed to walking gait by time of 27 second, and in amount time of 30 second, with duration of 7 seconds for transition, its gait is varied to trot gait. Similarly, adaption and evolution of parameters in the CPG are implemented by setting piecewise linear function with modulation signal. These piecewise linear functions are programmed depending to the value of modulation signal, CPG equations and amount of the transition time, which is defined the desired slope variation of the parameters in duration of the simulation. These coherent percepts for managing the desired Cartesian behavioral context is shown in (Figure 8). At summary, this methodology gives open-loop framework ability, in order to define the accurate timing of switching and synchronization of trajectories for landing position and time of the swing and stance feet in Cartesian space and consequently cause of spanning the motor skills for the quadruped robot.

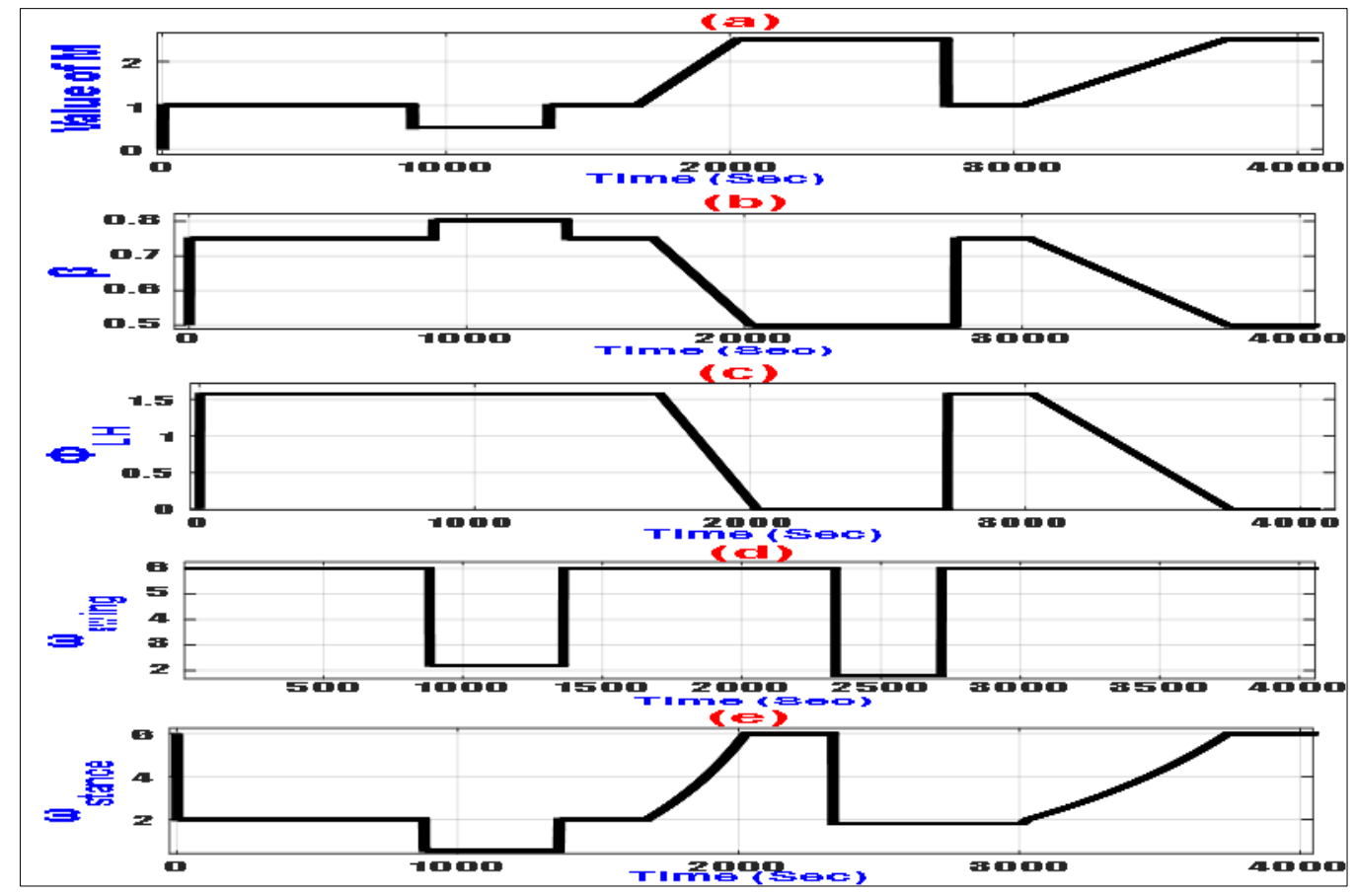

Figure 8 The excitation signal (a), variation and adjustment of duty factor (b), phase gait (c), (d) swing and (e) stance phase of the oscillators in $\mathrm{CPG}$

According to the obtained spatio-temporal vectors from the CPG, Cartesian footstep planner generates adaptable foot trajectories along the axis of movements for swing legs. So, through the footstep planner or Cartesian footstep planner at two stages, step length, step height, duration of swing and stance phases for each leg and relative phase between legs can be computed and adjusted. Moreover, due to the phase signals with precise phase relationship which is programmed by the modulation signal, gait transition, speed adjustment and phases between legs in Cartesian space are computed with reasonable accuracy. 

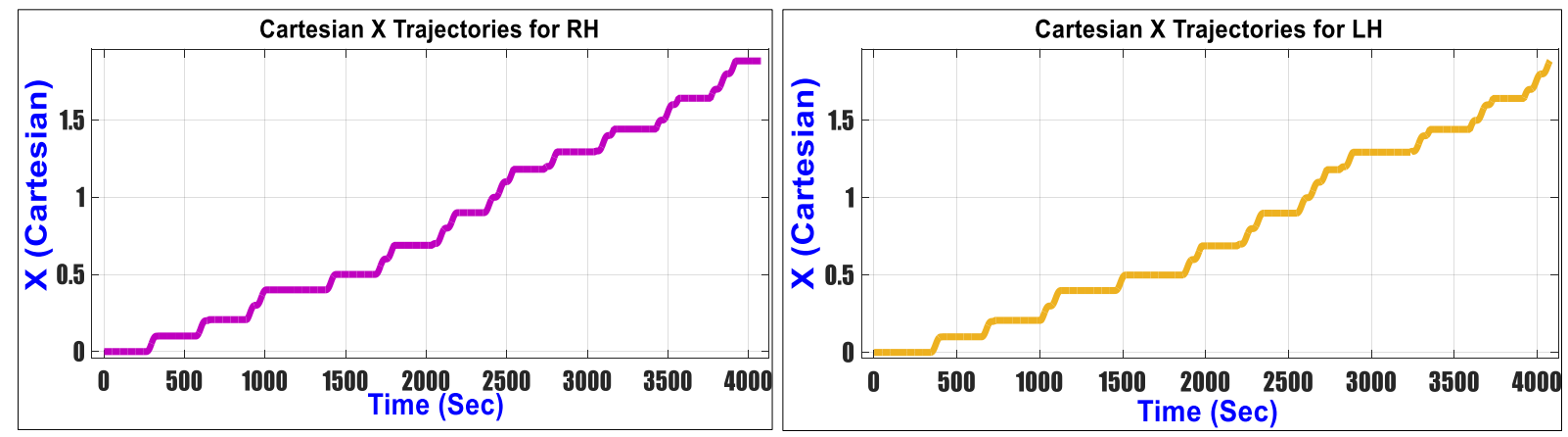

Figure 9a) Cartesian $X$ trajectories for RH Figure 9b) Cartesian $X$ trajectories for LH
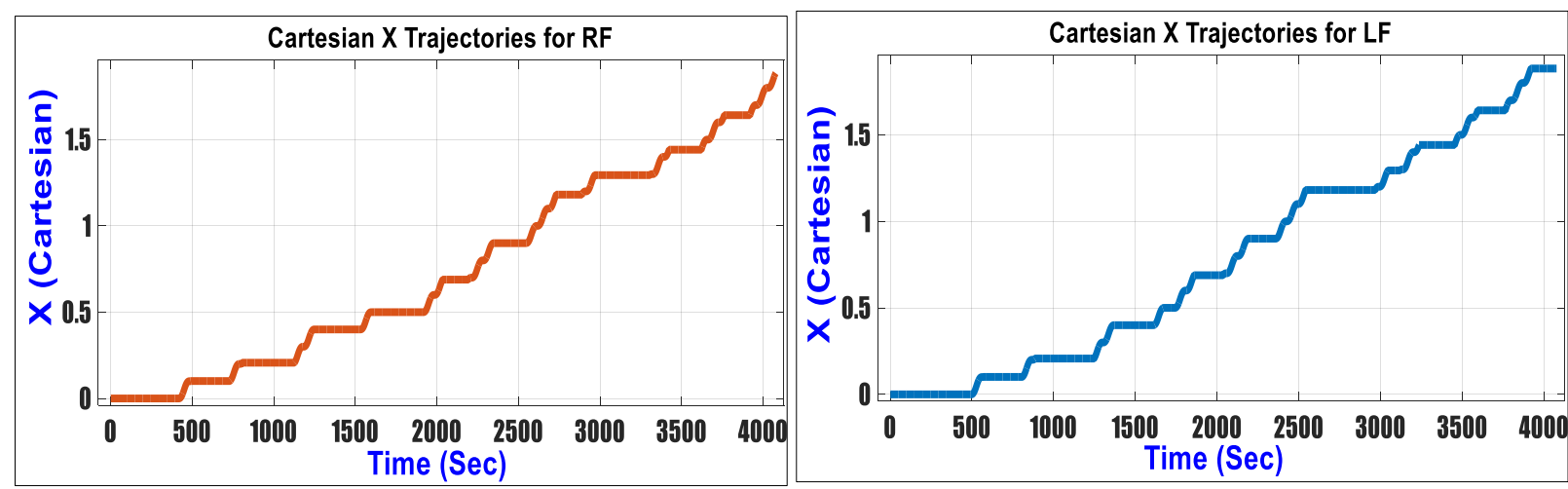

Figure 9c) Cartesian $X$ trajectories for RF Figure 9d) Cartesian $X$ trajectories for LF
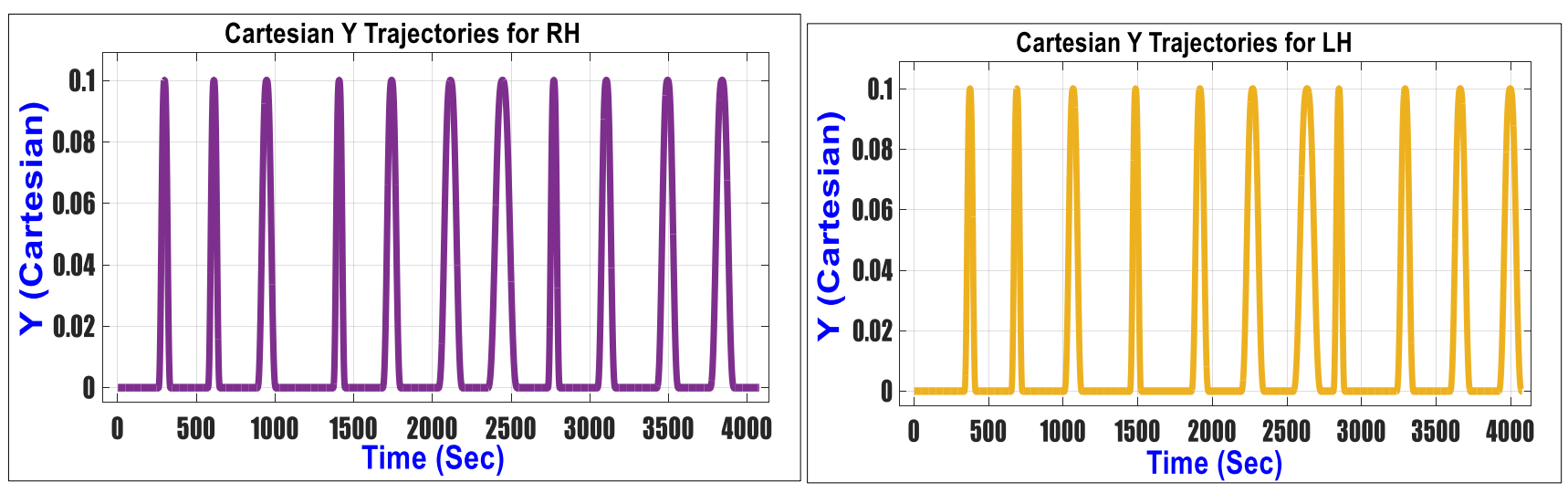

Figure 10a) Cartesian $Y$ trajectories for RH Figure 10b) Cartesian $Y$ trajectories for $\mathrm{LH}$
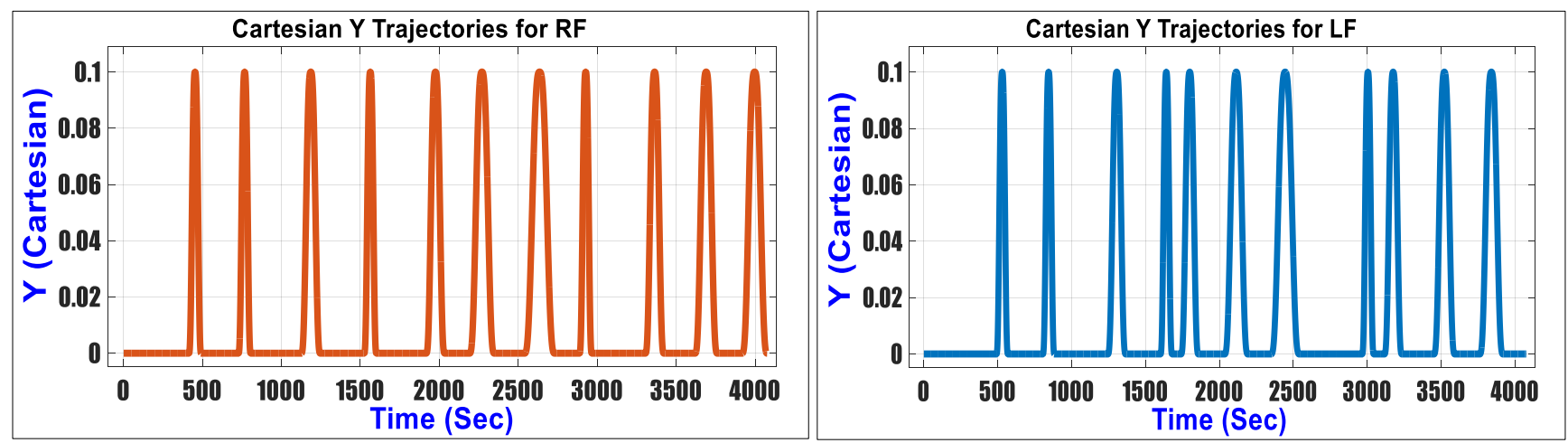

Figure 10c) Cartesian $Y$ trajectories for RF Figure 10d) Cartesian $Y$ trajectories for $L F$ 


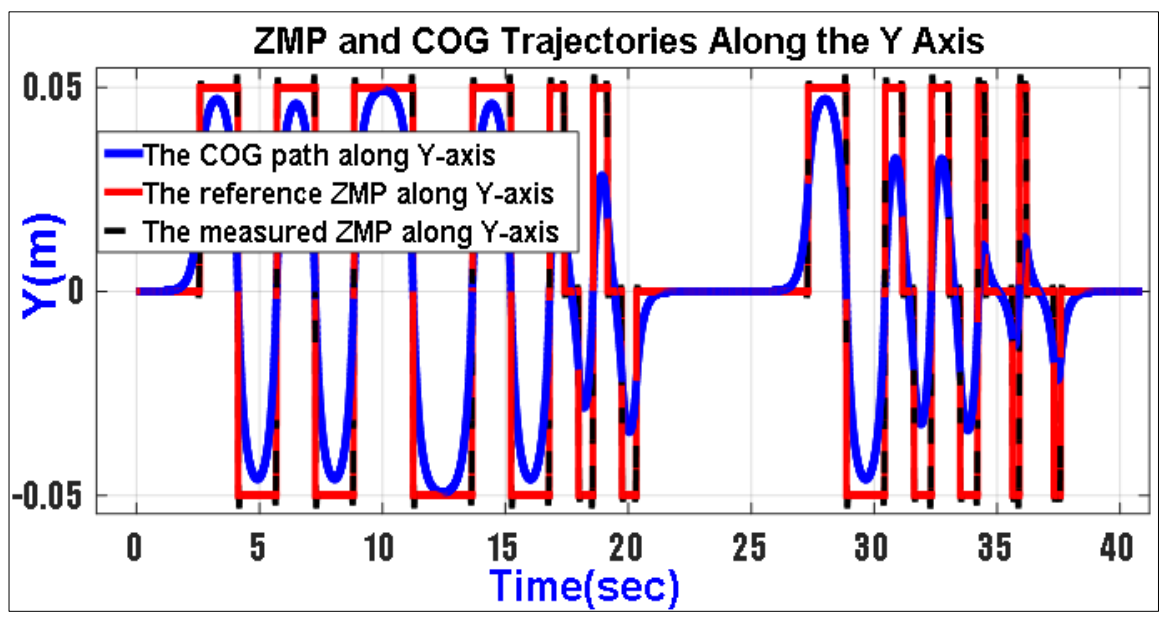

Figure 11a) COG and ZMP trajectories along the $Y$ axis

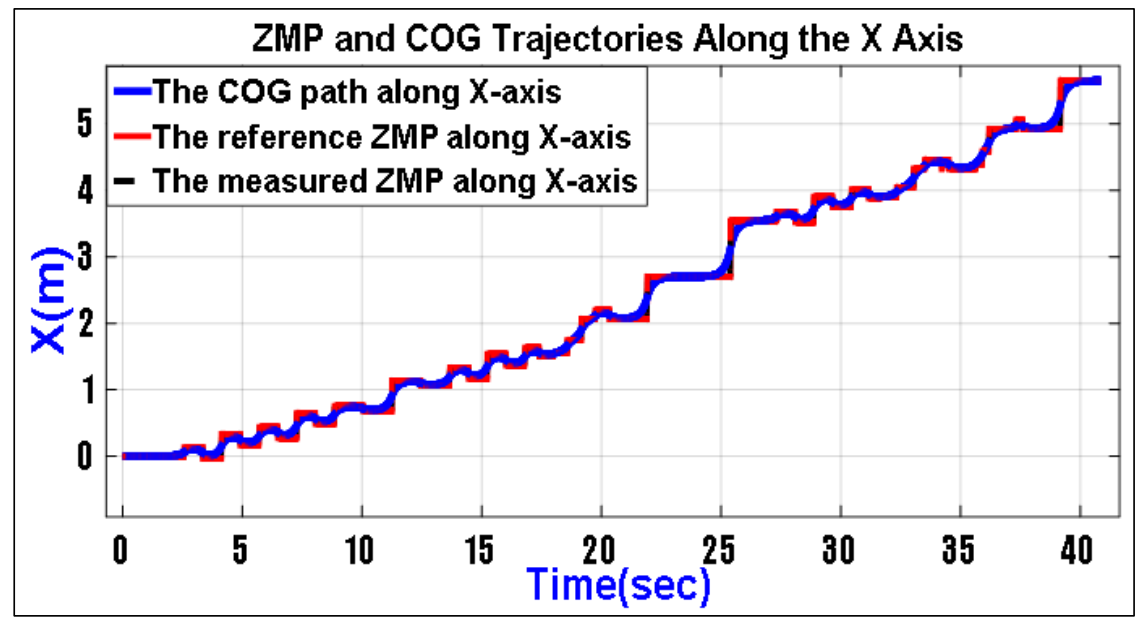

Figure 11b) Reference ZMP and desired ZMP and COG trajectories along the $\mathrm{X}$ axis of the robot

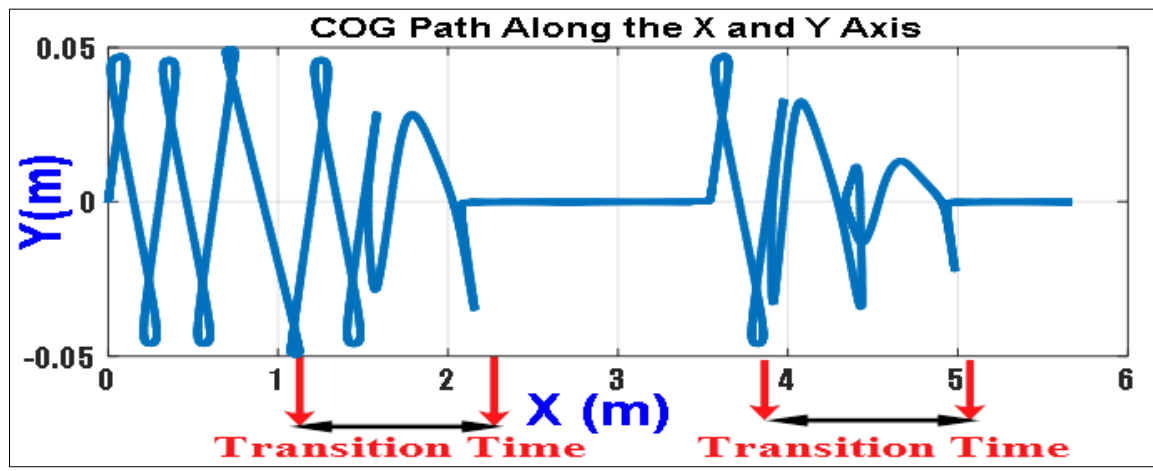

Figure 11c) COG path for the robot

Then, Cartesian foot planner are generated trajectories for tip of the swing legs along the $\mathrm{z}$ and $\mathrm{x}$ axis and its results are plotted in (Figure 9) and (Figure 10) respectively. Next, a reference ZMP trajectory based on the support polygon triangle of stance foots is obtained, and then desired ZMP trajectory is calculated with preview controller. Subsequently, COG trajectory is obtained, and its result is plotted in (Figure 11). Also, the related COG and ZMP trajectories along the y axis and the COG path are plotted in (Figure 11) accordingly. As a consequence, by using this approach, robot can switch smoothly, gradually and stably from different gait event sequence in the Cartesian space, and then these motor skills are mapped from workspace to joint space. Also, according to the excitation signal, this determination for the timing can be extended. 
In the literatures, the stability of the robot is defined by modulating the CPG parameters to produce coordinated joint control signal. In this way, many oscillators and consequently many parameters are required to control the joint space of the robot. In this paper, the coordination of the joints is done in the last step of the algorithm, and the CPG are utilized to generate and develop the motor skills of the robot in the Cartesian space. Then, by computing the inverse kinematics for the quadruped robot, joint variables trajectories could be obtained. Basically, this robot has 18 degrees of freedom which 6 degrees of freedom is for the body and others, 3 degree of freedom, are for each legs of the robot. These 3 degrees of freedom for each leg are hip flap, swing joints and knee joints. The joint trajectory for the hip flip joint of the leg LF is called q11, for hip swing q12, and for knee swing joint q13, and in this way others joint trajectories are defined for other legs $\{\mathrm{LH}=2, \mathrm{RF}=3, \mathrm{RH}=4\}$ respectively. By using the Inverse kinematic relationship for the robot, joint trajectories for all the 12 degrees of freedom of legs, in joint space, are computed and are ready for completing our experimental setup.

Joint trajectories for the hip and knee joints of the robot are plotted in (Figure 12a), and the range of the hip joints for the qi1 is [-180,180], and also in terms of (rad) for only the LF leg are plotted in (Figure 12b). These simulations commands are applied to the experimental robot, and then snapshot of the robot motion during the locomotion in different times, are plotted in (Figure 12c). In this algorithm, the assignment of foot step and height value for the legs are arbitrary in the section of the Cartesian footstep planner. So, according to the output power and torque of the DC servo motor, one can assign different values for these parameters.

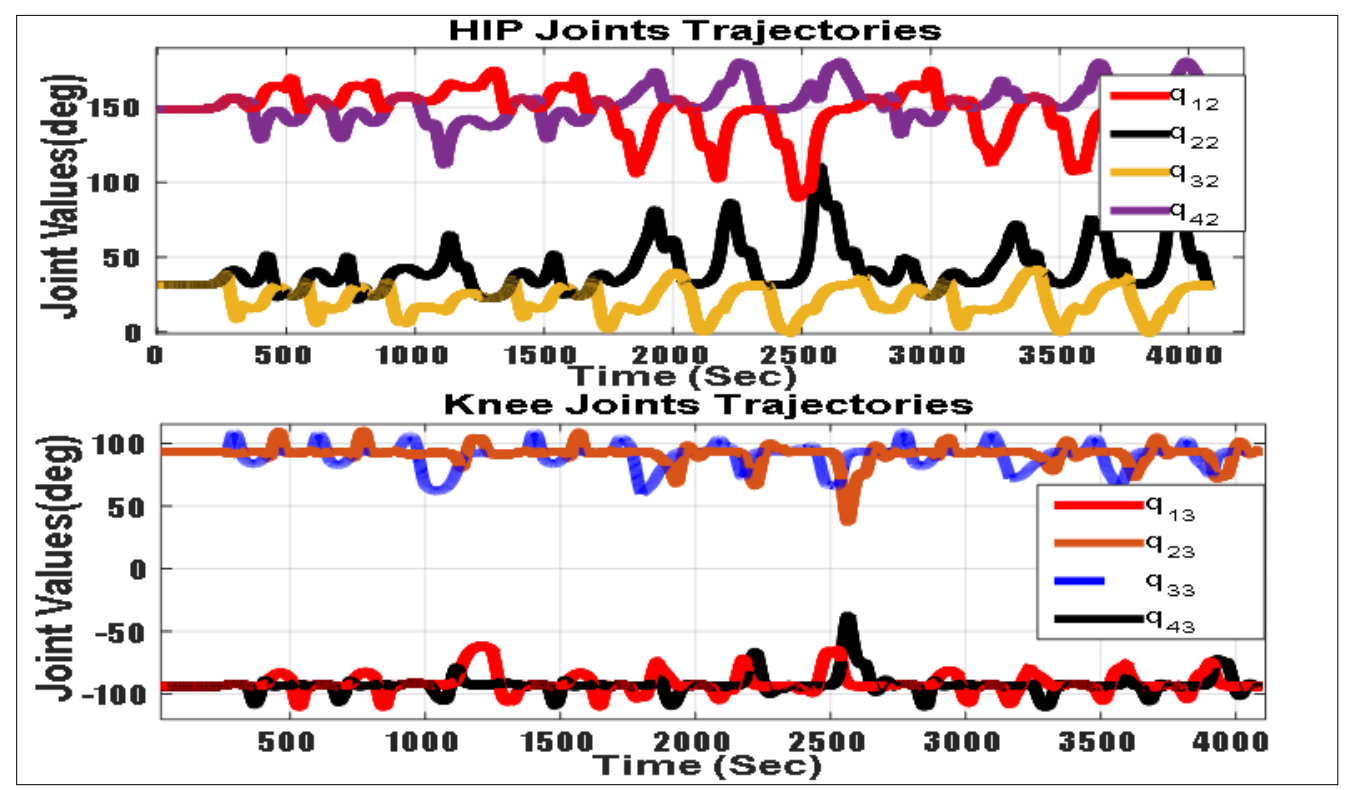

Figure 12a) Joint trajectories for hip and knee of the robot

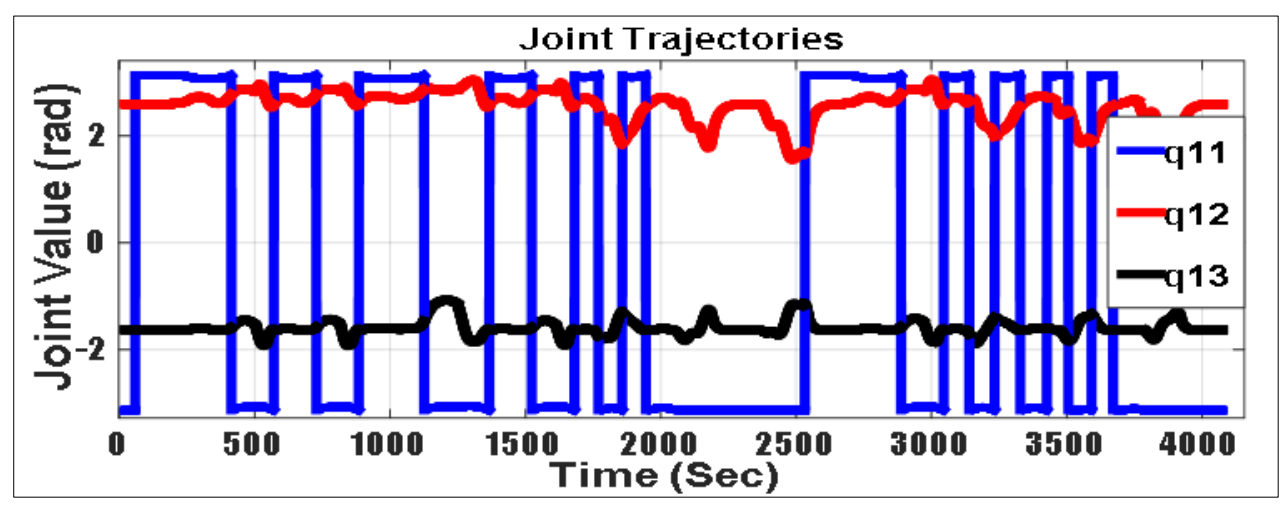

Figure 12b) Joint trajectories for LF leg of the robot 


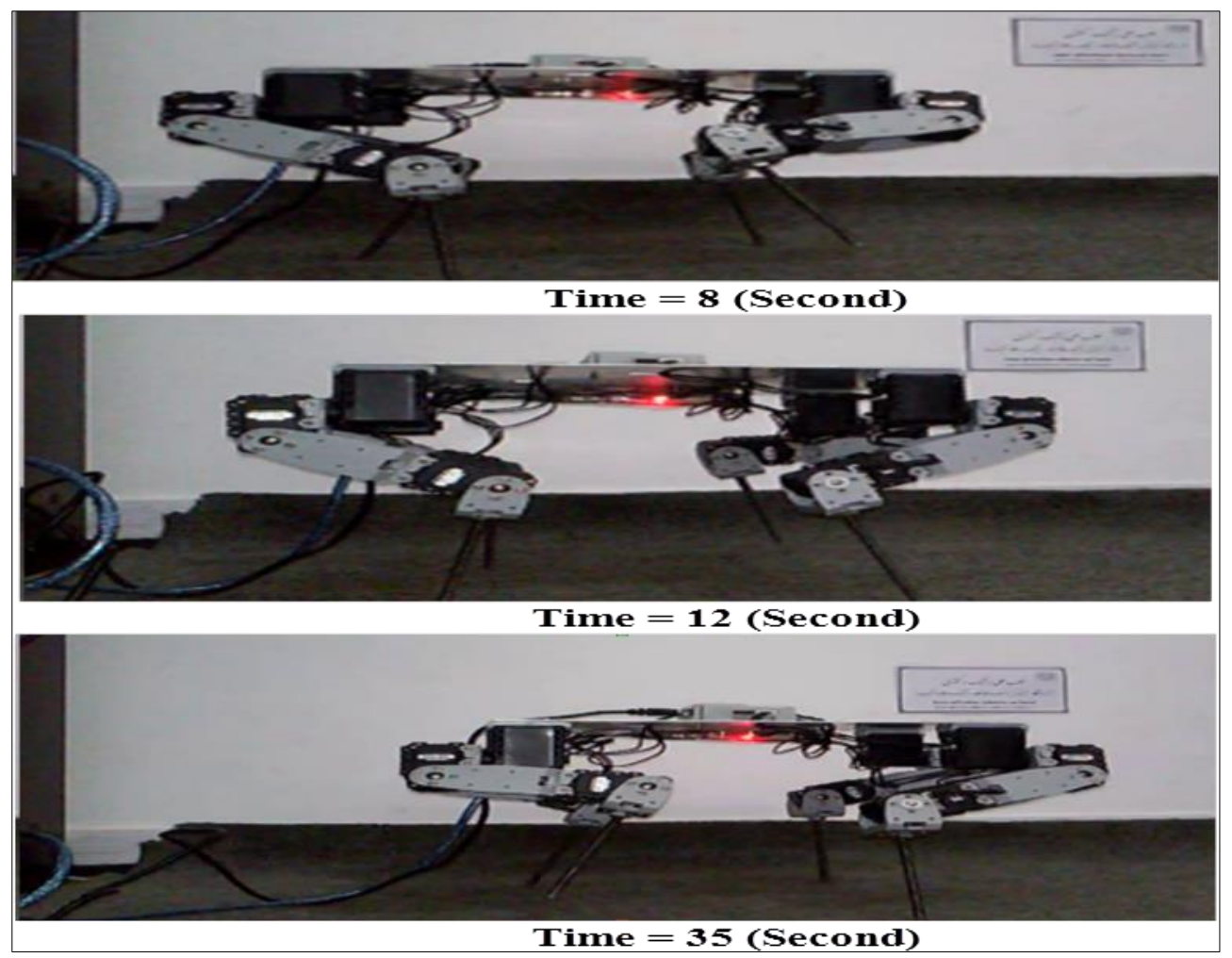

Figure 12c) Execution of simulations on the robot and capturing snapshot of the robot during the locomotion

\section{Discussion}

In this section, the introduced approaches of various references are implemented and compared to the proposed algorithm. Among the simulation results, the COG path of the robot which contains information about the smoothness of motion, synchronization and accuracy of coordination, is chosen for comparison with other references. Firstly, the previous simulated COG path, in (Figure 11c), are compared with some references and secondly, for clarifying more desirable features of the algorithm, simple walking to trot gait transition is simulated, and then its COG path is compared with other references. The references [33, 36 and 39] used matsouka oscillators and matrix coefficient approach in order to couple the oscillators of the CPG. Also, the CPG patterns are applied for encoding the joint trajectories of the robot in the joint space directly. Besides, harmonic functions are added to CPG equations in order to exist smooth transient motion between the gait switching. By applying their approaches, as shown in (Figure 13),

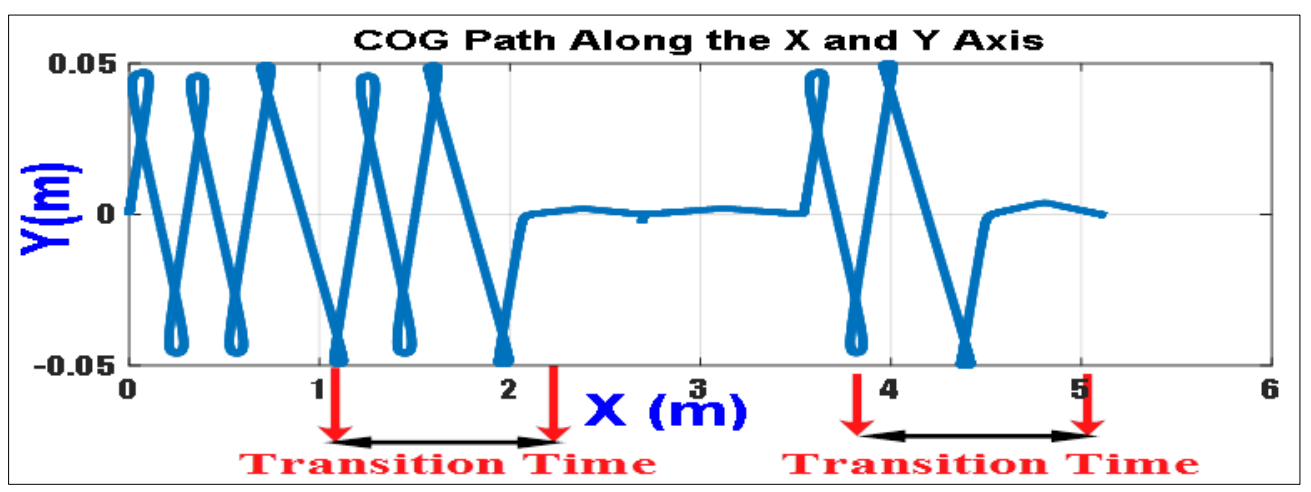

Figure 13 Comparison of COG path in the Figure 11c) with other references [33, 36 and 39]

It is not possible to reach to the gradual transition between different gaits, and only ability to change and control the stance phases of the feet can be achievable. Conversely, in our algorithm, all the vital parameters of the gait event sequences can be programmed with regard to the excitation signal. Furthermore, the modulation of stance or swing phases or its periods can be controlled independently and as well as the time of the transition. 
Generally, in order to attain to gradual gait transition, gait coordination algorithm is responsible for proper inter-leg coordination and smoothly and predictably adapts the motor patterns according to the commands. The references [1112], generated different gaits of the quadruped robot in the joint space with matrix coefficient approach. In these references, it cannot possible to appoint any time duration for condition of gait transition, so consequently, the robot is changed its gait suddenly at one predefined time. Alternatively, references like [13-17] utilized approach of coefficient matrix for each gait with ability to control the transition time, but any predefined setting function for directing or synchronizing the evolution of patterns between the gaits or adjusting parameters of the CPG are not existed. Finally, references like [20-21] used approaches which has only ability to adjust the duty factor of the gait in duration of the gait transition and generate different gaits. In the proposed simulation for comparison, the gait of the robot is changed from walking to trot gait at the time of 1 seconds, and the transition time is set as 1.5 seconds, and simulated results are shown in (Figure 14). According to the result, our algorithm has ability to program in the head of the time accurately and subsequently desired adjustment of the gait parameters like duty factor, phase gait and coordinating these parameters with each other and also step height, step foot and so on can be managed. Therefore, in this research, the ability to manage gait parameters and gait event sequences, and accordingly extraction of spatio-temporal vectors, are developed precisely. In this way, the development of behavioral diversity can be reached compared to earlier CPG-based works, which usually are used in the joint space, and enable us for enhancing the behavior for gait switching for the robot.

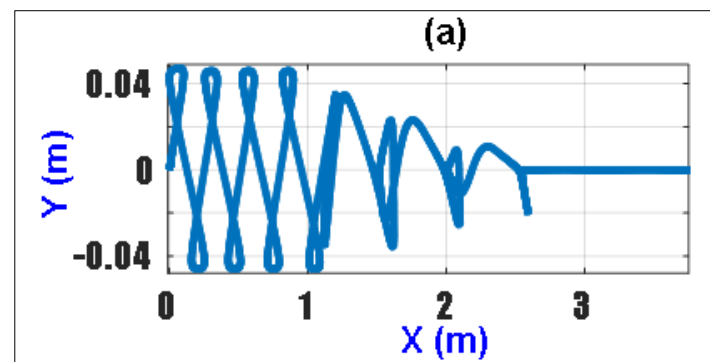

(d)

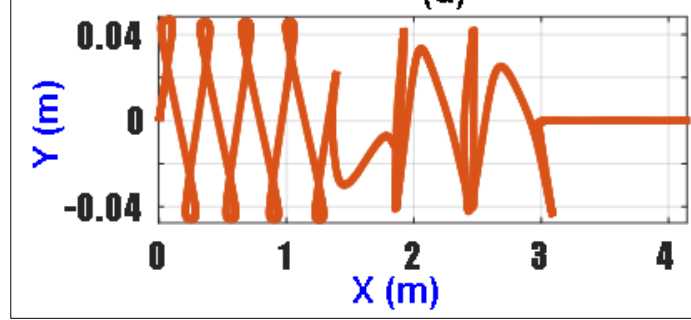

(b)

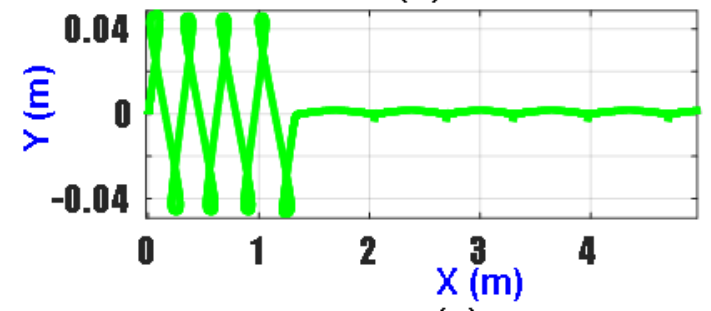

(c)

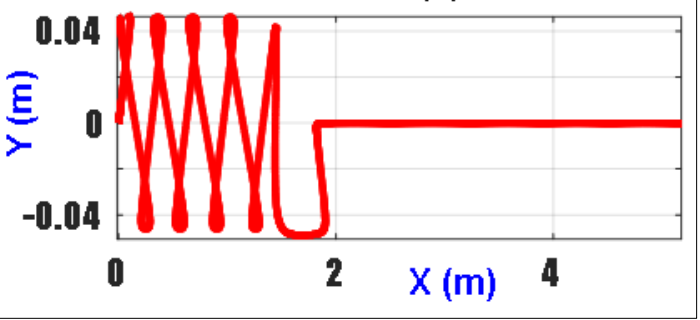

Figure 14 Comparison of the COG path with other references. This paper (a), Reference [11, 12] (b), Reference [1317] (c), Reference $[20,21](d)$

\section{Conclusion}

In this paper, a network of modified HOPF oscillators with ability of functional programming and extraction of the spatio-temporal vectors from the gait event sequences are developed. Then, by mapping the spatio-temporal vectors to Cartesian footstep basis function and by designing the preview controller, different desired Cartesian behavioral context can be managed for the robot. The simulation results are implemented on the quadruped robot and also its results are compared with other references for clarifying the contributions of the research. In summary, this algorithm can handle management of the necessary parameters space for creation of different adjustable gait and motor tasks for the quadruped robot. In addition, the speed, coordination between feet and many desirable features for locomotion can be programmed accurately and predictably. Also, in the future works by applying the structure of the proposed algorithm, it is possible to implement different locomotion scenarios for changing the foot step location in respect to the predefined Cartesian task or in order to cross the planar obstacles.

\section{Compliance with ethical standards}

\section{Acknowledgments}

Thanks to the Department of Mechanical Engineering, K. N. Toosi University of Technology, Center of Excellence in Robotics and Control, Advanced Robotics \& Automated Systems (ARAS) Lab Affiliation, Tehran, Iran. 


\section{Disclosure of conflict of interest}

All authors declare that they have no conflict of interest.

\section{References}

[1] Ijspeert AJ. Central pattern generators for locomotion control in animals and robots: a review. Neural networks. 2008 May 1; 21(4):642-53.

[2] Yang K. Dynamic model and CPG network generation of the underwater self-reconfigurable robot. Advanced Robotics. 2016 Jul 17; 30(14):925-37.

[3] Asadi F, Mollakazemi MJ, Uzelac IL, Moosavian SA. A novel method for arterial blood pressure pulse detection based on a new coupling strategy and discrete wavelet transform. In2015 Computing in Cardiology Conference (CinC) 2015 Sep 6 (pp. 1081-1084). IEEE.

[4] Yao C, Liu C, Xia L, Liu M, Chen Q. Humanoid adaptive locomotion control through a bioinspired CPG-based controller. Robotica. 2021:1-8.

[5] Bal C. Neural coupled central pattern generator based smooth gait transition of a biomimetic hexapod robot. Neurocomputing. 2021 Jan 8; 420:210-26.

[6] Asadi F, Mollakazemi MJ, Ghiasi S, Sadati SH. Enhancement of life-threatening arrhythmia discrimination in the intensive care unit with morphological features and interval feature extraction via random forest classifier. In2016 Computing in Cardiology Conference (CinC) 2016 Sep 11 (pp. 57-60). IEEE.

[7] Yazdani M, Salarieh H, Foumani MS. Bio-inspired decentralized architecture for walking of a 5-link biped robot with compliant knee joints. International Journal of Control, Automation and Systems. 2018 Dec; 16(6):2935-47.

[8] Asadi F, Mollakazemi MJ, Atyabi SA, Uzelac IL, Ghaffari A. Cardiac arrhythmia recognition with robust discrete wavelet-based and geometrical feature extraction via classifiers of SVM and MLP-BP and PNN neural networks. In2015 Computing in Cardiology Conference (CinC) 2015 Sep 6 (pp. 933-936). IEEE.

[9] Tamura D, Aoi S, Funato T, Fujiki S, Senda K, Tsuchiya K. Contribution of phase resetting to adaptive rhythm control in human walking based on the phase response curves of a neuromusculoskeletal model. Frontiers in neuroscience. 2020 Feb 5; 14:17.

[10] Li J, Wang J, Yang SX, Zhou K, Tang H. Gait planning and stability control of a quadruped robot. Computational intelligence and neuroscience. 2016 Mar; 2016.

[11] Rutishauser S, Sprowitz A, Righetti L, Ijspeert AJ. Passive compliant quadruped robot using central pattern generators for locomotion control. In2008 2nd IEEE RAS \& EMBS International Conference on Biomedical Robotics and Biomechatronics 2008 Oct 19 (pp. 710-715). IEEE.

[12] Righetti L, Ijspeert AJ. Pattern generators with sensory feedback for the control of quadruped locomotion. In2008 IEEE International Conference on Robotics and Automation 2008 May 19 (pp. 819-824). IEEE.

[13] Barron-Zambrano JH, Torres-Huitzil C, Girau B. Configurable embedded CPG-based control for robot locomotion. International Journal of Advanced Robotic Systems. 2012 Sep 28;9(3):92.

[14] Lara-Nino CA, Torres-Huitzil C, Barron-Zambrano JH. Versatile educational and research robotic platform based on reconfigurable hardware. In2014 International Conference on ReConFigurable Computing and FPGAs (ReConFig14) 2014 Dec 8 (pp. 1-6). IEEE.

[15] Barron-Zambrano JH, Torres-Huitzil C, Girau B. Perception-driven adaptive CPG-based locomotion for hexapod robots. Neurocomputing. 2015 Dec 25; 170:63-78.

[16] Barron-Zambrano JH, Torres-Huitzil C. CPG Implementations for Robot Locomotion: Analysis and Design, A. Robotic Systems-Applications, Control and Programming. 2012 Feb 3:1-23.

[17] Barron-Zambrano JH, Torres-Huitzil C, Girau B. Hardware implementation of a CPG-based locomotion control for quadruped robots. InInternational Conference on Artificial Neural Networks 2010 Sep 15 (pp. 276-285). Springer, Berlin, Heidelberg.

[18] Buchli J, Righetti L, Ijspeert AJ. Engineering entrainment and adaptation in limit cycle systems. Biological Cybernetics. 2006 Dec; 95(6):645-64. 
[19] Shim Y, Husbands P. Chaotic exploration and learning of locomotion behaviors. Neural computation. 2012 Aug 1; 24(8):2185-222.

[20] Owaki D, Morikawa L, Ishiguro A. Gait transition of quadruped robot without interlimb neural connections. Dynamic Walking 2012. 2012.

[21] Tsuchiya K, Aoi S, Tsujita K. Locomotion control of a biped locomotion robot using nonlinear oscillators. InProceedings 2003 IEEE/RSJ International Conference on Intelligent Robots and Systems (IROS 2003)(Cat. No. 03CH37453) 2003 Oct 27 (Vol. 2, pp. 1745-1750). IEEE.

[22] Asadi F, Khorram M, Moosavian SA. CPG-based gait transition of a quadruped robot. In2015 3rd RSI International Conference on Robotics and Mechatronics (ICROM) 2015 Oct 7 (pp. 210-215). IEEE.

[23] Asadi F, Khorram M, Moosavian SA. CPG-based gait planning of a quadruped robot for crossing obstacles. In2015 3rd RSI International Conference on Robotics and Mechatronics (ICROM) 2015 Oct 7 (pp. 216-222). IEEE.

[24] Matos V, Santos CP. Towards goal-directed biped locomotion: Combining CPGs and motion primitives. Robotics and Autonomous Systems. 2014 Dec 1; 62(12):1669-90.

[25] Ma Z, Liang Y, Tian H. Research on Gait Planning Algorithm of Quadruped Robot Based on Central Pattern Generator. In2020 39th Chinese Control Conference (CCC) 2020 Jul 27 (pp. 3948-3953). IEEE.

[26] Yue J. Learning Locomotion For Legged Robots Based on Reinforcement Learning: A Survey. In2020 International Conference on Electrical Engineering and Control Technologies (CEECT) 2020 Dec 10 (pp. 1-7). IEEE.

[27] Yu H, Gao H, Deng Z. Enhancing adaptability with local reactive behaviors for hexapod walking robot via sensory feedback integrated central pattern generator. Robotics and Autonomous Systems. 2020 Feb 1; 124:103401.

[28] Niwa T, Amano Y, Jimbo T. Bio-inspired cooperative control using a nonlinear oscillator without direct communication among agents. In2020 59th Annual Conference of the Society of Instrument and Control Engineers of Japan (SICE) 2020 Sep 23 (pp. 501-506). IEEE.

[29] Maufroy C, Kimura H, Takase K. Integration of posture and rhythmic motion controls in quadrupedal dynamic walking using phase modulations based on leg loading/unloading. Autonomous Robots. 2010 Apr; 28(3):331-53.

[30] Zamani A, Khorram M, Moosavian SA. Dynamics and stable gait planning of a quadruped robot. In2011 11th International Conference on Control, Automation and Systems 2011 Oct 26 (pp. 25-30). IEEE.

[31] Khorram M, Moosavian SA. Stable walking of a quadruped robot on uneven terrains with friction constraints. In2014 Second RSI/ISM International Conference on Robotics and Mechatronics (ICRoM) 2014 Oct 15 (pp. 364369). IEEE.

[32] Saputra AA, Botzheim J, Ijspeert AJ, Kubota N. Combining Reflexes and External Sensory Information in a Neuromusculoskeletal Model to Control a Quadruped Robot. IEEE Transactions on Cybernetics. 2021 Feb 26.

[33] Gu S, Meng F, Liu B, He Z, Fan X, Ming A, Huang Q. Gait Transition Method for Quadruped Robot Based on CPG Network and Impedance Control. In2020 5th International Conference on Advanced Robotics and Mechatronics (ICARM) 2020 Dec 18 (pp. 387-391). IEEE.

[34] Khorram M, Moosavian SA. A 3D stable trot of a quadruped robot over uneven terrains. Proceedings of the Institution of Mechanical Engineers, Part C: Journal of Mechanical Engineering Science. 2017 Feb; 231(3):55573.

[35] Khorram M, Moosavian SA. Push recovery of a quadruped robot on challenging terrains. Robotica. 2017 Aug; 35(8):1670-89.

[36] Guan X, Zheng H, Zhang X. Biologically inspired quadruped robot biosbot: modeling, simulation and experiment. In2nd International Conference on Autonomous Robots and Agents 2004 Dec (pp. 261-266).

[37] Katayama T, Ohki T, Inoue T, Kato T. Design of an optimal controller for a discrete-time system subject to previewable demand. International Journal of Control. 1985 Mar 1; 41(3):677-99.

[38] Kajita S, Kanehiro F, Kaneko K, Fujiwara K, Harada K, Yokoi K, Hirukawa H. Biped walking pattern generation by using preview control of zero-moment point. In2003 IEEE International Conference on Robotics and Automation (Cat. No. 03CH37422) 2003 Sep 14 (Vol. 2, pp. 1620-1626). IEEE.

[39] Liyao Z, Haojun Z, Xiuli Z, Zhifeng C. Realization of the biologically-inspired dynamic walking controller of a quadruped robot. InIEEE International Conference on Robotics, Intelligent Systems and Signal Processing, 2003. Proceedings. 20032003 Oct 8 (Vol. 2, pp. 937-941). IEEE. 